\title{
Late Quaternary Explosive Volcanic Activities of the Mindanao-Molucca Sea Collision Zone in the Western Pacific as Inferred from Marine Tephrostratigraphy in the Celebes Sea
}

\author{
Yueh-Ping $\mathrm{Ku}^{1,2}$, Chang-Hwa Chen ${ }^{3, *}$, Sheng-Rong Song ${ }^{2}$, \\ Yoshiyuki Iizuka ${ }^{3}$, and Jason Jiun-San Shen ${ }^{3}$ \\ ${ }^{1}$ Institute of Environmental Engineering, National Chiao Tung University, Hsinchu, Taiwan, ROC \\ 2 Department of Geosciences, National Taiwan University, Taipei, Taiwan, ROC \\ ${ }^{3}$ Institute of Earth Sciences, Academia Sinica, Taipei, Taiwan, ROC
}

Received 26 March 2008, accepted 30 July 2008

\begin{abstract}
The giant piston core MD01-2387 taken from the eastern Celebes Sea basin provides a marine tephrostratigraphy with high resolution for inferring explosive volcanism in the area surrounding the basin for the past $350 \mathrm{kyr}$. The sequence contains 65 tephra layers composed of volcanic minerals and glassy particles. The compositional characteristics of crystal enriched tephra layers and the ${ }^{87} \mathrm{Sr} /{ }^{86} \mathrm{Sr}$ of the glass particles ( 0.7039 to 0.7042$)$ suggest that these ashes were released by the volcanoes in the Mindanao-Molucca Sea collision zone, including the south Philippine Arc system, the Sangehi Arc and the Halmahera Arc. The upward decrease of tephra layers in this core indicates a declining trend of explosive volcanic activity of this collision zone, particularly, after ca. $180 \mathrm{ka}$ in the late Middle Pleistocene. This record also suggests that the shoshonitic volcanism in Central Mindanao became extinct at ca. $151 \mathrm{ka}$, which is much younger than what reported previously by onland investigations.
\end{abstract}

Key words: Tephra, Volcanic activity, Celebes Sea

Citation: Ku, Y. P., C. H. Chen, S. R. Song, Y. Iizuka, and J. J. S. Shen, 2009: Late quaternary explosive volcanic activities of the Mindanao-Molucca Sea collision zone in the western pacific as inferred from marine tephrostratigraphy in the Celebes Sea. Terr. Atmos. Ocean. Sci., 20, 587-605, doi: 10.3319/TAO.2008.07.30.01(TT)

\section{INTRODUCTION}

Discrete marine tephra layers represent instant ash fallout deposits because volcanic materials sink more rapidly than other pelagic or hemipelagic particles (Wiesner et al. 1995). Over time, stratified tephra layers preserved in deep-sea basins are better protected against external erosive forces than those preserved on land. Thus, the deep-sea tephrostratigraphic record can provide a complete record of large volcanic eruptions in a particular area. Marine microfossils in the intervenient intervals provide biostratigraphic correlation markers by which the record can be well tied to global chronology.

In 2001, a giant piston core for IMAGES (International

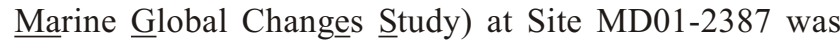
raised from the $\underline{\text { Celebes }} \underline{\text { Sea }} \underline{B} a \sin (\mathrm{CSB})$ at $4^{\circ} 47.27^{\prime} \mathrm{N}$ latitude and $123^{\circ} 30.09^{\prime} \mathrm{E}$ longitude (Fig. 1) and is very close to the ODP Site $767 \mathrm{~B}\left(4^{\circ} 47.47^{\prime} \mathrm{N}\right.$ in latitude and $123^{\circ} 30.20^{\prime} \mathrm{E}$

\footnotetext{
* Corresponding author

E-mail: china@earth.sinica.edu.tw
}

in longitude). The age of Core MD01-2387 was estimated to be younger than $460 \mathrm{ka}$ (Bassinot and Baltzer 2002). The sediments of Core MD01-237 with well preserved discrete ash layers offer a new and more complete record to examine and construct the late Quaternary explosive volcanic activity surrounding the CSB.

The CSB is 4500 - $5500 \mathrm{~m}$ deep, and is located at the complex junction of three major converging plates: the Eurasian plate to the west, the Philippine Sea plate to the east, and the Australian plate to the south (Fig. 1). Consequently, the CSB is surrounded by Islands made of Eocene-Quaternary igneous rocks (Pubellier et al. 1991; Hall 1997). In fact, volcanism is still active on some of the islands (Simkin and Siebert 1994).

Results from previous studies of the deep-sea ash sequence at ODP (ㅁcean Drilling Program) Site 767 (Pubellier et al. 1991) and onland volcanics dating (Sajona et al. 1997) indicate that the active period of volcanism surrounding the CSB reflects the geodynamic adjustments of plate conver- 


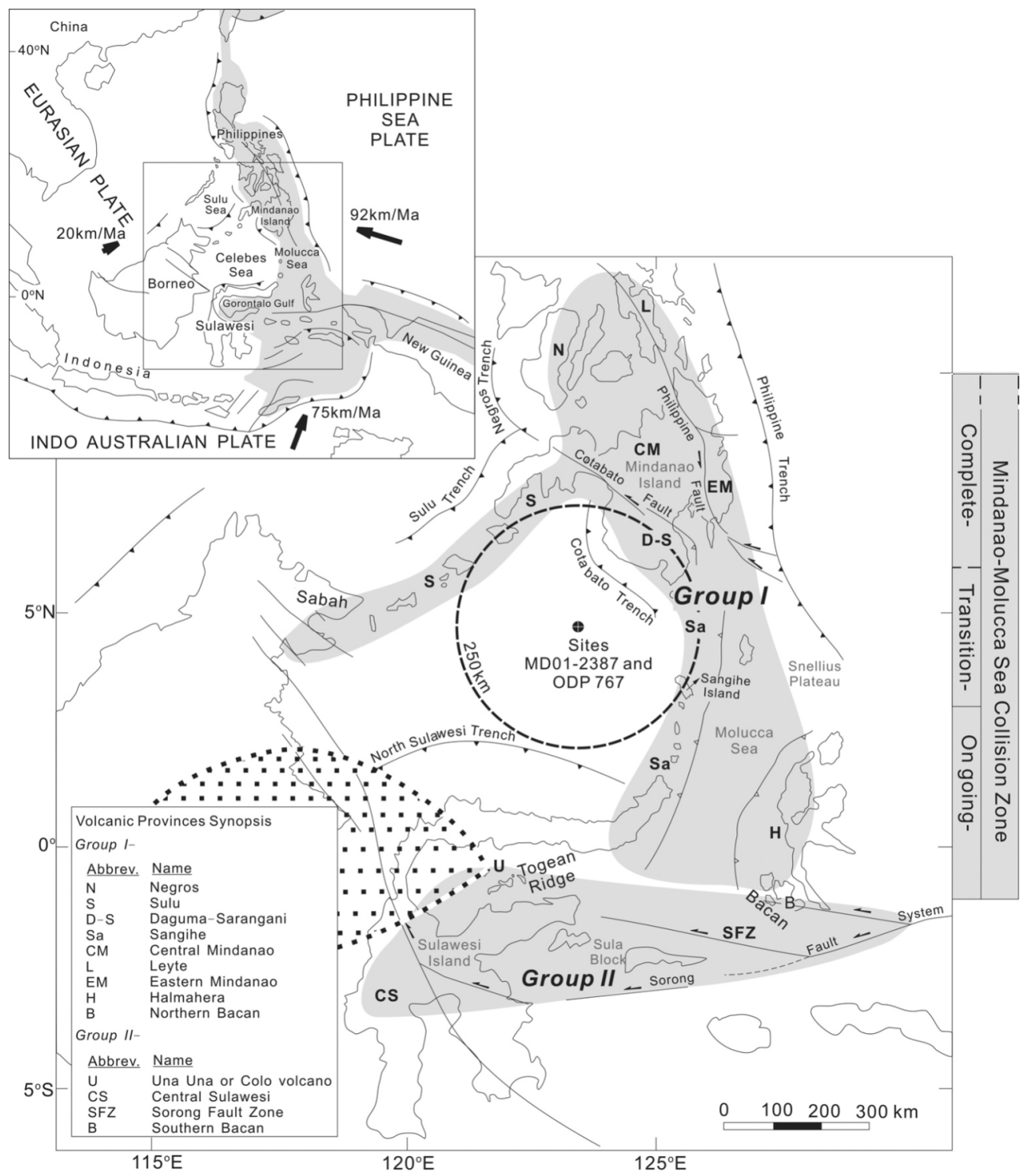

Fig. 1. Tectonic setting and Quaternary volcanic provinces surrounding the Celebes Sea Basin (Morrice et al. 1983; Pubellier et al. 1991; Delfin et al. 1997; Moss et al. 1998; Elburg and Foden 1999). Shaded area of the left top panel represents the tectonic boundary between the three major plates (Rangin 1991); the arrows with numbers represent the direction of plate motion and speed (Hinschberger et al. 2005). Volcanic provinces are grouped into two groups by locale. Please see the text for a description of the $250-\mathrm{km}$ radius circle around the coring site enclosed by the dashed lines. The ash covered area of the 1898 eruption of the Una Una Volcano (Wichmann 1902) is marked by the dotted area. The division of the Mindanao-Molucca Sea collision zone follows Moore and Silver (1983).

gence in the aforementioned plates. Via the closure of the Molucca Sea basin, plate convergence has resulted in the welding of two arcs, the Eastern Mindanao Arc (the northern extension of the Hamahera Arc) and the Daguma-Sarangani Arc (the northern extension of the Sangihe Arc), at Mindanao Island after $5 \mathrm{Ma}$ (Fig. 1) and leads the subduction systems surrounding this island to be active. A last important active period of volcanism surrounding the CSB took place at around 2.5 Ma (Pubellier et al. 1991).

The convergence of the three major plates is still active, and the welding of the two arcs has extended and migrated southward. At present, the closure of the Molucca Sea formed a collision zone in the Mindanao Island and the Molucca Sea, called the Mindanao-Molucca Sea collision zone (Moore and Silver 1983) (Fig. 1). This collision zone has been subdivided as the complete-, transition-, and on going-collision zones from Mindanao Island down to the southern part of the Molucca Sea (Moore and Silver 1983). Studies of onland volcanic rocks (e.g., Baker and Malaihollo 1996; Delfin et al. 1997; Sajona et al. 1997; Elburg and Foden 1998) indicate that paleo-explosive volcanism has changed spatially and temporally. Combining the published results of onland investigations with the record of deep-sea tephras should result in a more robust history of explosive volcanism in the area. 


\section{VOLCANIC PROVINCES AND TECTONIC SETTING}

The CSB is surrounded by extensive Quaternary volcanics and many active Holocene volcanoes (Simkin and Siebert 1994), considered to be the sources of the marine tephra layers found in Core MD01-2387 (Fig. 1). The scattered distribution of the Quaternary volcanics, with an identifiable regional trench-subduction system and tectonic frame, has been classified into several Quaternary volcanic provinces as summarized below (Sajona et al. 1997; Polvé et al. 2001; Macpherson et al. 2003).

In the northern CSB, the Oligocene/Early Miocene Sulu Sea crust subducts eastward and southward along the Negros and Sulu Trenches to form the Negros and Sulu Arcs, respectively (Sajona et al. 2000a; Castillo et al. 2002; Solidum et al. 2003). The Sulu Arc extends from northwestern Sabah (Tongkul 1991) into the western peninsula of Mindanao Island. There are three other volcanic provinces in the Mindanao Island: (1) the Daguma-Sarangani Arc (or Cotabato Arc) in the western part of Mindanao Island, associated with the eastward subduction of the Eocene Celebes Sea crust along the Cotabato Trench (Sajona et al. 1997; Sajona and Maury 1998); (2) the Eastern Mindanao Arc (or Pacific Cordillera Arc) in the eastern part of Mindanao Island, associated with the westward subduction of the Philippine Sea plate along the Philippine Trench (Sajona et al. 1997; Sajona and Maury 1998); and (3) the Central Mindanao Area, which is restricted to the area between the Philippine Fault and the Cotabato Fault and is associated with a detached remnant of the Molucca Sea plate under Mindanao Island (Sajona et al. 1994, 1997, 2000b). It appears that the Quaternary volcanics of the Central Mindanao Area are not related to any subduction system. Instead, they are considered to be a post-collision volcanic system (Sajona et al. 1994, 1997, 2000b). The Philippine Sea plate subducts westward along the Philippine Trench to form the Leyte Arc to the north of Mindanao Island (Sajona et al. 1997). To the south of Mindanao Island, the westward subduction of the Molucca Sea plate (Macpherson et al. 2003) forms the Sangihe Arc to the west. This arc extends from the southern end of Mindanao Island, through the Sangihe Island, and then, into the northeast arm of Sulawesi Island. The Halmahera Arc to the east passes southward through Bacan Island, which is a fragment of the continental crust (Morris et al. 1983). At the southern end of the Molucca Sea plate, a number of small extinct volcanic islands lie along the Sorong Fault Zone (Morris et al. 1983). To the south of the CSB, the Una Una Volcano area might relate to the southward subduction of the Eocene Celebes Sea crust along the North Sulawesi Trench (Katili and Sudradjat 1984; Kopp et al. 1999). The Quaternary volcanics in the Central Sulawesi area do not appear to be related to any subduction system, but are considered to have been formed by the post-col- lisional melting of continental crust (Priadi et al. 1994; Polvé et al. 2001).

In general, these complicated volcanic provinces can be categorized into two major groups: Group I and II volcanic zones (Fig. 1). The tectonic settings and volcanic activities of these two groups are summarized below.

\subsection{Group I Volcanic Zone}

The Group I volcanic zone includes nine volcanic provinces located in the northern and eastern CSB (Fig. 1). Quaternary magmatism of these volcanic provinces is related to the closure of the Molucca Sea. As it closes, the Molucca Sea crust subducts on both sides forming the Sangihe Arc and the Halmahera Arc as well as the northern portion of Bacan Island in the west and east. The closure of the Molucca Sea has resulted in the formation of an on-going collision zone in the southern Molucca Sea. In addition, the closure forms a complete-collision zone beneath Mindanao Island, via the welding of the northern extensions of the two arcs, the Sangihe and Halmahera Arcs. The fusion of the two arcs has reinitiated several subductions around Mindanao Island (Pubellier et al. 1991; Sajona et al. 1997; Pubellier et al. 1999). It has also generated two types of volcanic provinces: one along the Negros, Sulu, Daguma-Sarangani, Leyte, and Eastern Mindanao Arcs related to the now reactive subductions; and another in the Central Mindanao Area (i.e., post-collision volcanic province), which is related to a detached remnant of the Molucca Sea plate beneath it. Both results of onland investigations and deep-sea records indicate that the last important volcanic activity of the Group I volcanic zone began ca. 2.5 Ma (Pubellier et al. 1991; Sajona et al. 1997). Studies of onland volcanic rocks (e.g., Baker and Malaihollo 1996; Sajona et al. 1997) indicate that the Quaternary explosive volcanism in this area has changed spatially and temporally.

\subsection{Group II Volcanic Zone}

Quaternary volcanic activity in the Group II volcanic zone is not related to the closure of the Molucca Sea basin. The Group II volcanic zone includes four volcanic areas: (1) southern Bacan Island; (2) Sorong Fault Zone in the southern Molucca Sea; (3) the Central Sulawesi Area; and (4) Volcano Una Una in the southern part of the Celebes Sea (Fig. 1). The southern portion of the Molucca Sea plate terminates at the left-lateral strike slip of the Sorong Fault system, which has been the major tectonic boundary of the Philippine plate and the Australian plate for the past $13 \mathrm{Ma}$ (Hinschberger et al. 2005). A number of small extinct volcanic islands, which have no available dates but are thought to be of late Tertiary (Morris et al. 1983), are found along the Sorong Fault Zone. At present the left-lateral strike slip fault system extends across Bacan Island and into Sulawesi. It 
also forced the Sula block to collide westward with Sulawesi at around $5 \mathrm{Ma}$ (Hinschberger et al. 2005). The westward collision between the Sula block and Sulawesi initiated the southward subduction of Celebes Sea crust along the North Sulawesi Trench, which is related to the activity of Volcano Una Una (Kopp et al. 1999).

\section{MATERIALS AND METHODS}

The Core MD01-2387 is $32.88 \mathrm{~m}$ in length and raised from a depth of $4866 \mathrm{~m}$. It is primarily composed of clay with ash spots, intercalated by tens of ash layers (Bassinot and Baltzer 2002).

The ash layers were examined by observing the texture of the splitting core's surface and the constitution of the ash layers under stereomicroscope. The thickness or stratigraphic intervenient depth for each discrete ash layer was identified by apparent changes in color and particle size of the sediment profile from the surface of the splitting core. One cubic centimeter of sediments of each ash layer was collected. The samples were processed by ultrasonic bath and wet sieving. The constitution and morphology of the particles coarser than $63 \mu \mathrm{m}$ were further recognized under stereomicroscope. Volcanic minerals and glass particles were then identified from 65 layers.

The 65 discrete tephra layers were further classified into crystal-rich layers (27 layers) and glass-rich layers (38 layers) according to their different dominant particle constituents. The dominant ( $>50$ vol.\%) particle constituents (volcanic mineral crystals and glass particles) were examined under a stereomicroscope. The glass particles of each discrete tephra layer were further described by their color, particle shape (morphology), and vesicle shape (texture). Thus, the glass-rich layers were further grouped into pale-glass-rich layers (28 layers) and dark-glass-rich layers (10 layers).

The chemical and $\mathrm{Sr}$ isotopic compositions of the glass particles of the 22 glass-rich tephra layers were analyzed. Twelve pale-glass-rich layers, each over ca. $2.5 \mathrm{~cm}$ thick, and seven dark-glass-rich layers, each over ca. $1.0 \mathrm{~cm}$ thick, were selected for chemical composition analysis. Three glass-rich layers preserved in depths shallower than $1120 \mathrm{~cm}$ were also selected, but for major element chemical composition analysis only.

The major element contents of the glass particles, 7 15 grains, were determined for each analyzed layer using a JEOL JXA-8900R EMPA at the Institute of Earth Sciences, Academia Sinica in Taipei. Concentrations of 9 major elements ( $\mathrm{Si}, \mathrm{Ti}, \mathrm{Al}, \mathrm{Fe}, \mathrm{Mn}, \mathrm{Mg}, \mathrm{Ca}, \mathrm{Na}$, and $\mathrm{K}$ ) were analyzed under the following operating conditions: $10 \mathrm{kV}$, $5 \mathrm{nA}$ and focused $(3 \mu \mathrm{m})$ for accelerated voltage, probe current, and beam diameter on polished vesicular glass particles. The data were processed by the ZAF [atomic number $(\underline{Z}), \underline{A}$ bsorption and Fluorecense] correction rou- tine. Data with a total oxide value of less than 95 wt.\% were excluded. The strontium element of the glass particles was extracted using a Sr-Spec column from ca. 500 to 2000 glass particles for each analyzed layer. The strontium isotopic ratios of the glass particles were measured by a Finnigan-MAT 262 TIMS at the same institute in Taipei. The Sr isotopic ratios were normalized to ${ }^{86} \mathrm{Sr} /{ }^{88} \mathrm{Sr}=$ 0.1194 given a mean of $0.710319 \pm 0.000037(2 \sigma)$ for ${ }^{87} \mathrm{Sr} /{ }^{86} \mathrm{Sr}$ of the NBS-987 Sr.

\section{RESULTS}

\subsection{Occurrence of Tephra Layers}

A total of 65 discrete tephra layers were recognized by microscopic examination. The number of layers in the upper portion $(<1800 \mathrm{~cm})$ is clearly less than that in the lower part $(>1800 \mathrm{~cm})$ (Table 1 and Fig. 2). Meanwhile, the thickness of the tephra layers varies from a few millimeters to $160 \mathrm{mil}-$ limeters (Table 1 and Fig. 5b).

\subsection{Nature of the Particles}

\subsubsection{Compositions}

These tephra layers were further classified into either crystal-rich layers (27/65 layers) or glass-rich layers (38/65 layers) (Table 1) depending on their dominant (> 50 vol.\%) composition of particles. The predominant types of mineral crystals in the crystal-rich layer and the predominant types of glass particles in the glass-rich layer are shown in Table 1. The former consists of rectangular and euhedral crystals, such as single plagioclase and clino-pyroxene grains with subsidiary ortho-pyroxene and biotite. The remaining components $(<50$ vol. $\%)$ are vesicular glass particles, mostly pale in color. In the glass-rich layers, however, the morphology and texture of the predominantly glass particles vary with color, ranging from dark to pale (Table 1). The dark-glass particles often possess irregular-grain morphology and spherical-vesicle texture. The pale-glass particles, in contrast, tend to have rod-like or platy-grain morphology and elongate-vesicle texture. Thus, the glass-rich layers (38/65 layers) can be readily further classified into dark-glass-rich layers (10/38 layers) and pale-glass-rich layers (28/38 layers) (Table 1 and Fig. 2). The remaining components in the glass-rich layers are volcanic crystals; such as, clino-pyroxene and plagioclase with subsidiary biotite.

Figure 2 presents the distribution of three types of tephra layers in the core. The crystal-rich layers decrease significantly in depths shallower than $1900 \mathrm{~cm}$. The darkglass-rich layers disappear entirely at depths shallower than $1400 \mathrm{~cm}$. The interval between $1975-3288 \mathrm{~cm}$ possesses more crystal-rich layers than that between $0-1975 \mathrm{~cm}$. For the dark-glass-rich layers, the last occurrence is at the depth of ca. $1444 \mathrm{~cm}$ (Table 1 and Fig. 2). 
Table 1. Physical description and silicate content of each discrete ash layer in Core MD01-2387.

\begin{tabular}{|c|c|c|c|c|c|c|c|c|c|c|c|c|}
\hline \multirow{5}{*}{$\begin{array}{c}65 \\
\text { Layers }\end{array}$} & \multirow{5}{*}{$\begin{array}{c}\text { Core } \\
\text { Section }^{1}\end{array}$} & \multirow{5}{*}{$\begin{array}{l}\text { Depth }(\mathrm{cm}) \text { of } \\
\text { Discrete Layer } \\
\text { (or Spot) Base }\end{array}$} & \multirow{5}{*}{\begin{tabular}{|c|} 
Layer \\
Thickness \\
$(\mathrm{cm})$
\end{tabular}} & \multicolumn{9}{|c|}{ Predominant Constitutions (> 50 vol. $\%$ ) } \\
\hline & & & & \multirow{4}{*}{\begin{tabular}{|c|}
$\begin{array}{c}\text { Crystal-rich } \\
\text { layers }\end{array}$ \\
\end{tabular}} & \multicolumn{7}{|c|}{ Glass-rich layers } & \multirow{4}{*}{$\begin{array}{c}\text { Depth } \\
\text { Interval } \\
\text { (cm) }\end{array}$} \\
\hline & & & & & \multicolumn{5}{|c|}{ Glass type $^{3}$} & \multirow{3}{*}{$\begin{array}{c}\text { Silicate } \\
\text { Content }^{4} \\
\text { of Glasses } \\
(w t \%)\end{array}$} & \multirow{3}{*}{$\begin{array}{l}\text { Layer } \\
\text { Type }^{5}\end{array}$} & \\
\hline & & & & & \multicolumn{3}{|c|}{ Dark-glass-rich layers } & \multicolumn{2}{|c|}{$\begin{array}{c}\text { Pale-glass-rich } \\
\text { layers }\end{array}$} & & & \\
\hline & & & & & $\begin{array}{c}\text { brown- } \\
\text { (dark) }\end{array}$ & $\begin{array}{c}\text { light } \\
\text { brown- }\end{array}$ & gray- & white- & $\begin{array}{c}\text { colorless- } \\
\text { (pale) }\end{array}$ & & & \\
\hline \multirow{21}{*}{ 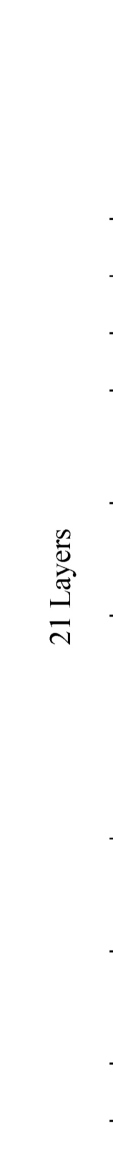 } & II & 164.0 & 4.0 & & & & & $\mathrm{r}$ & & $78.73 \pm 0.33$ & A & $161-162$ \\
\hline & & 190.0 & 2.5 & & & & & $\mathrm{r}$ & $\mathrm{r}$ & $78.79 \pm 0.66$ & A & $188-189$ \\
\hline & & 258.0 & 0.8 & plag, cpx & & & & & & & & $257-258$ \\
\hline & & 282.0 & - & plag, cpx & & & & & & & & $281-282$ \\
\hline & III & 352.0 & 2.0 & & & & & $\mathrm{r}$ & & $79.53 \pm 0.40$ & A & $350-351$ \\
\hline & IV & 503.0 & 1.0 & & & & & $\mathrm{r}$ & & $79.73 \pm 0.45$ & A & $501-502$ \\
\hline & $\mathrm{V}$ & 670.0 & 10.0 & & & & & & d & $75.27 \pm 0.73$ & A & $667-668$ \\
\hline & VI & 807.0 & 2.0 & plag, cpx & & & & & & & & $805-806$ \\
\hline & & 877.0 & 2.0 & plag, cpx & & & & & & & & $876-877$ \\
\hline & VII & 1002.0 & $<0.5$ & plag, cpx & & & & & & & & $1001-1002$ \\
\hline & & 1031.0 & $<0.5$ & plag, cpx & & & & & & & & $1030-1031$ \\
\hline & VIII & 1065.0 & 2.0 & & & & & $\mathrm{r}$ & & & & $1065-1066$ \\
\hline & & 1082.5 & 3.0 & & & & & $\mathrm{r}$ & & $79.31 \pm 0.56$ & A & $1080-1081$ \\
\hline & & 1121.0 & 1.0 & & & & & $\mathrm{r}$ & & $79.64 \pm 0.35$ & A & $1120-1121$ \\
\hline & & 1134.0 & $<0.5$ & plag, cpx & & & & & & & & $1133-1134$ \\
\hline & IX & 1241.0 & 5.0 & & & & & $\mathrm{p}$ & $\mathrm{p}$ & $77.30 \pm 0.52$ & A & $1238-1239$ \\
\hline & & 1271.0 & $<0.5$ & & & & & $\mathrm{r}$ & & & & $1270-1271$ \\
\hline & $\mathrm{X}$ & 1446.0 & 2.0 & & & $\mathrm{r}$ & $\mathrm{r}$ & & & $61.36 \pm 0.66$ & B & $1444-1445$ \\
\hline & & 1467.0 & - & plag, cpx & & & & & & & & $1466-1467$ \\
\hline & XI & 1583.5 & 5.0 & & & & & $\mathrm{p}$ & $\mathrm{p}$ & $76.40 \pm 0.47$ & A & $1580-1581$ \\
\hline & XII & 1757.0 & 16.0 & & & & & & $\mathrm{i}$ & $79.28 \pm 0.56$ & A & $1747-1748$ \\
\hline \multirow{12}{*}{ 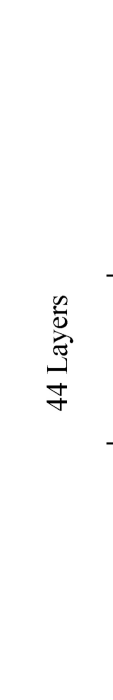 } & XIII & 1824.0 & 6.0 & & & & & $\mathrm{r}$ & & $80.14 \pm 0.43$ & A & $1822-1823$ \\
\hline & & 1834.0 & 2.0 & & & & & $\mathrm{r}$ & & & & $1832-1833$ \\
\hline & & 1883.0 & 1.0 & & & & & & $\mathrm{r}$ & & & $1882-1883$ \\
\hline & & 1904.0 & 4.0 & & & & & $\mathrm{r}$ & & $78.96 \pm 0.65$ & A & $1902-1903$ \\
\hline & & 1932.0 & - & & & & & $\mathrm{r}$ & & & & 1931-1932 \\
\hline & XIV & 1961.0 & $<0.5$ & & & & & $\mathrm{r}$ & & & & $1960-1961$ \\
\hline & & 1975.0 & $<0.5$ & plag, cpx, opx & & & & & & & & $1974-1975$ \\
\hline & & 2001.0 & 0.5 & plag, cpx, opx & & & & & & & & $2000-2001$ \\
\hline & $X V$ & 2111.0 & 1.0 & & & & & $\mathrm{r}$ & & & & $2110-2111$ \\
\hline & & 2130.0 & 1.0 & & & & & $\mathrm{r}$ & & & & $2129-2130$ \\
\hline & & 2208.5 & 0.5 & plag, cpx, opx & & & & & & & & 2208-2209 \\
\hline & & 2225.5 & 0.5 & $\begin{array}{l}\text { plag, cpx, } \\
\text { opx, bt }\end{array}$ & & & & & & & & $2225-2226$ \\
\hline
\end{tabular}


Table 1. (Continued).

\begin{tabular}{|c|c|c|c|c|c|c|c|c|c|c|c|c|}
\hline \multirow{5}{*}{$\begin{array}{c}65 \\
\text { Layers }\end{array}$} & \multirow{5}{*}{$\begin{array}{c}\text { Core } \\
\text { Section }^{1}\end{array}$} & \multirow{5}{*}{$\begin{array}{l}\text { Depth }(\mathrm{cm}) \text { of } \\
\text { Discrete Layer } \\
\text { (or Spot) Base }\end{array}$} & \multirow{5}{*}{$\begin{array}{c}\text { Layer } \\
\text { Thickness } \\
\text { (cm) }\end{array}$} & \multicolumn{9}{|c|}{ Predominant Constitutions ( $>50$ vol. $\%$ ) } \\
\hline & & & & \multirow{4}{*}{$\begin{array}{l}\text { Crystal-rich } \\
\text { layers }\end{array}$} & \multicolumn{7}{|c|}{ Glass-rich layers } & \multirow{4}{*}{$\begin{array}{c}\text { Depth } \\
\text { Interval } \\
\text { (cm) }\end{array}$} \\
\hline & & & & & \multicolumn{5}{|c|}{ Glass type $^{3}$} & \multirow{3}{*}{$\begin{array}{c}\begin{array}{c}\text { Silicate } \\
\text { Content }^{4}\end{array} \\
\begin{array}{c}\text { of Glasses } \\
(w t \%)\end{array}\end{array}$} & \multirow{3}{*}{$\begin{array}{l}\text { Layer } \\
\text { Type }^{5}\end{array}$} & \\
\hline & & & & & \multicolumn{3}{|c|}{ Dark-glass-rich layers } & \multicolumn{2}{|c|}{$\begin{array}{c}\text { Pale-glass-rich } \\
\text { layers }\end{array}$} & & & \\
\hline & & & & & \begin{tabular}{|c|} 
brown- \\
(dark)
\end{tabular} & $\begin{array}{c}\text { light } \\
\text { brown- }\end{array}$ & gray- & white- & $\begin{array}{c}\text { colorless- } \\
\text { (pale) }\end{array}$ & & & \\
\hline \multirow{32}{*}{ 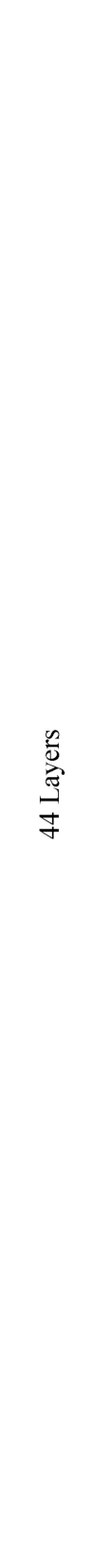 } & XVI & 2270.0 & 1.0 & & $\mathrm{r}$ & & & & & $56.74 \pm 1.53$ & B & $2269-2270$ \\
\hline & & 2352.0 & 5.5 & & & $\mathrm{p}$ & & & & $73.58 \pm 0.48$ & A & $2346-2347$ \\
\hline & & 2368.3 & 1.1 & plag, cpx & & & & & & & & $2368-2369$ \\
\hline & & 2377.0 & - & plag, cpx & & & & & & & & $2376-2377$ \\
\hline & XVII & 2431.0 & 0.5 & plag, cpx & & & & & & & & $2430-2431$ \\
\hline & & 2484.0 & 1.0 & plag, cpx & & & & & & & & $2483-2484$ \\
\hline & & 2499.0 & 2.0 & plag, cpx & & & & & & & & $2497-2498$ \\
\hline & & 2532.5 & 8.5 & & & $\mathrm{i}$ & & & & $62.19 \pm 1.51$ & B & $2531-2532$ \\
\hline & & 2544.0 & 2.0 & & & & & & $\mathrm{~d}$ & & & $2542-2544$ \\
\hline & XVIII & 2583.0 & 2.0 & & $\mathrm{i}$ & & $\mathrm{i}$ & & & $59.27 \pm 1.96$ & B & $2582-2583$ \\
\hline & & 2638.0 & 1.5 & plag, cpx & & & & & & & & $2637-2638$ \\
\hline & & 2673.0 & 11.0 & & & & & $\mathrm{r}$ & & $77.99 \pm 0.63$ & A & $2672-2673$ \\
\hline & & 2692.0 & 2.5 & & & & & $\mathrm{r}$ & & & & $2690-2691$ \\
\hline & XIX & 2732.0 & $<0.5$ & plag, cpx & & & & & & & & $2731-2732$ \\
\hline & & 2745.0 & $<0.5$ & plag, cpx, opx & & & & & & & & $2744-2745$ \\
\hline & & 2800.7 & 0.6 & plag, cpx, opx & & & & & & & & $2800-2801$ \\
\hline & & 2821.0 & 0.5 & plag, cpx & & & & & & & & $2820-2821$ \\
\hline & & 2839.5 & 1.7 & & $\mathrm{i}$ & & & & & $58.98 \pm 0.79$ & B & $2839-2840$ \\
\hline & XX & 2866.5 & 0.5 & plag, opx & & & & & & & & $2866-2867$ \\
\hline & & 2914.0 & 4.0 & & & & & $\mathrm{p}$ & & $76.05 \pm 1.02$ & A & 2913-2914 \\
\hline & & 2922.0 & 2.0 & & & & & $\mathrm{r}$ & & & & 2921-2922 \\
\hline & & 2939.0 & 5.5 & & & & & $\mathrm{r}$ & & $78.34 \pm 0.93$ & A & 2933-2934 \\
\hline & XXI & 3022.0 & 4.0 & & & $\mathrm{~d}$ & & & & $53.37 \pm 1.84$ & $\mathrm{C}$ & $3019-3020$ \\
\hline & & 3039.0 & $<0.5$ & plag, cpx & & & & & & & & $3038-3039$ \\
\hline & & 3053.1 & 1.2 & & & & & $\mathrm{r}$ & & & & $3048-3054$ \\
\hline & & 3079.0 & $<0.5$ & plag, cpx & & & & & & & & $3078-3079$ \\
\hline & & 3097.0 & $<0.5$ & plag, cpx, bt & & & & & & & & 3096-3097 \\
\hline & & 3114.5 & 0.6 & & & $\mathrm{i}$ & & & & & & $3114-3115$ \\
\hline & & 3137.5 & 0.5 & plag, cpx & & & & & & & & $3137-3138$ \\
\hline & XXII & 3205.1 & 0.9 & & $\mathrm{i}$ & & & & & & & $3204-3205$ \\
\hline & & 3222.0 & 0.5 & & & & & $\mathrm{r}$ & & & & $3221-3222$ \\
\hline & & 3270.0 & 1.0 & & $\mathrm{i}$ & & & & & & & $3269-3270$ \\
\hline
\end{tabular}

Note: Corebase: $3288 \mathrm{~cm}$

1. The core is preserved and numbered by sections. Each section is $150 \mathrm{~cm}$ in length.

2. Crystal types - plag: plagioclase; cpx: clino-pyroxene; opx: ortho-pyroxene; bt: biotite.

3. Glass types - I: irregular clast with spherical vesicles; $r$ : rod-like clast with elongate vesicles; $p$ : platy clast with elongate vesicles; $d$ : disk-like clast with spherical vesicles.

4. Average value plus and minus 1 sd.

5. Types A to C were classified by the geochemistry property, please see the text. 
(a)

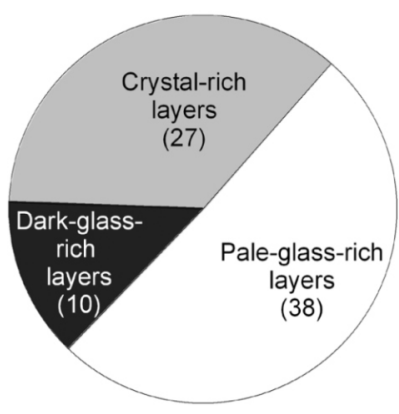

(b)

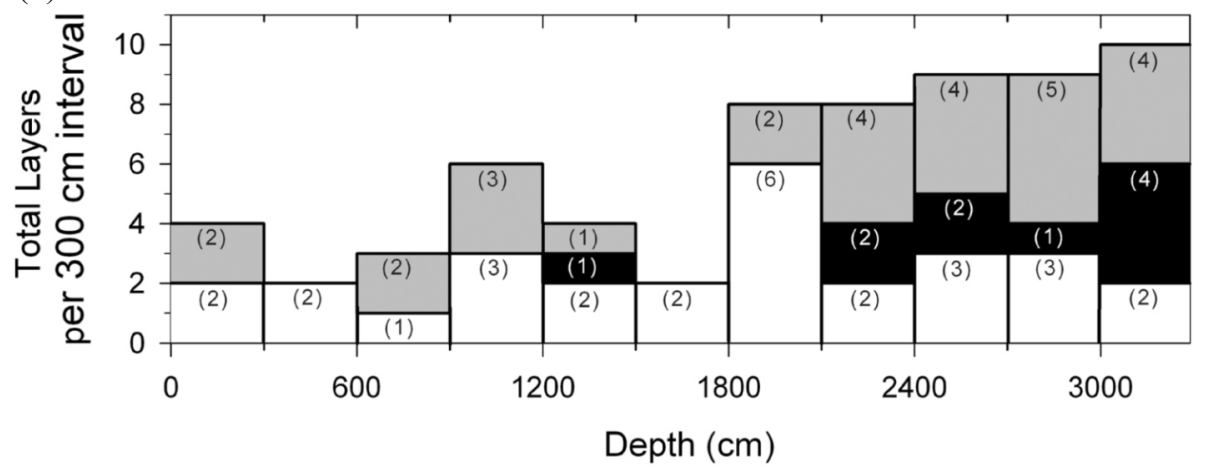

Fig. 2. (a) Pie chart showing numbers of three types for ash layers in Core MD01-2387. (b) Histograms of the tephra layers contributed from three types for ash layers at $300-\mathrm{cm}$ intervals.

\subsubsection{Geochemistry}

The glass particles of 22 glass-rich layers were subjected to chemical analyses (Tables 1,2). In terms of their chemical property these layers can be classified into three types: Types A, B, and $\mathrm{C}$, as recognized by their distribution on the biplot of the total content of alkalis $\left(\mathrm{Na}_{2} \mathrm{O}+\mathrm{K}_{2} \mathrm{O}\right)$ vs. $\mathrm{SiO}_{2}$ (Fig. 3a). The $\mathrm{Sr}$ isotopic ratios of glass particles in the 22 glass-rich layers range from 0.70376 to 0.70420 (Fig. 4, Table 2). Variation in $\mathrm{Sr}$ isotopic ratios of the glass particles is related to the Type (A, B, or C) of layer. Most pale-glass-rich layers belong to Type $\mathrm{A}$ but the dark-glass-rich layers belong to Type B or C. Generally, Type A has higher ${ }^{87} \mathrm{Sr} /{ }^{86} \mathrm{Sr}$ ratios $(>0.70389)$ than Types B and C $(<0.70389)$ (Fig. 4).

\section{Type A Glass-Rich Layers}

Type A glass particles are plotted in the rhyolitic field with $73.0-80.8$ wt.\% of $\mathrm{SiO}_{2}$ and $3.4-7.6$ wt.\% for $\mathrm{Na}_{2} \mathrm{O}+\mathrm{K}_{2} \mathrm{O}$ (Fig. 3a). They possess $\mathrm{Sr}$ isotopic ratios in the range of $0.70389-0.70420$ (Fig. 4). There are 16 Type A layers (Table 1) of which 15 are pale-glass-rich layers and the remaining one is a dark-glass-rich layer. The 16 Type A layers are distributed randomly throughout the core (Table 1, Fig. 2).

\section{Type B Glass-Rich Layers}

The glass particles of Type B layers possess a rela- tively high total alkalis content and lower silica content, as fall in the trachyandesitic and trachytic composition fields $\left(\mathrm{Na}_{2} \mathrm{O}+\mathrm{K}_{2} \mathrm{O}=6.1\right.$ - 10.1 wt.\%; $\mathrm{SiO}_{2}=54.7$ - 64.7 wt.\%) (Fig. 3a). They can also be classified into the high-K and shoshonite series in a $\mathrm{K}_{2} \mathrm{O}$ vs. $\mathrm{SiO}_{2}$ diagram (Taylor et al. 1981) (not shown here). The $\mathrm{Sr}$ isotopic ratios for Type $\mathrm{B}$ glass particles lie in a narrow range of $0.70376-0.70387$ (Fig. 4). Five dark-glass-rich layers comprise the Type B layers. The most recent occurrence of a Type B layer is recorded at the depth of ca. $1444 \mathrm{~cm}$ (Table 1 and Fig. 2b), which also marks the last occurrence of the dark-glass-rich layers.

\section{Type C Glass-Rich Layer}

Only one tephra layer contains glass particles of relatively low silica content and low total alkalis content. It falls in the basaltic and basaltic andesitic composition fields $\left(\mathrm{SiO}_{2}=50.6-55.8\right.$ wt.\%; $\mathrm{Na}_{2} \mathrm{O}+\mathrm{K}_{2} \mathrm{O}=3.1-5.2$ wt.\% $)$ (Fig. 3a and Table 1). The Sr isotopic ratio for the glass particles is 0.70383 (Fig. 4).

\section{DISCUSSION}

The erupted volcanic ashes, mainly glass shards, pumices and mineral crystals, are carried by prevailing winds, including the troposphere and lower stratosphere wind 
Table 2. Geochemical analysis results of glass particles from IMAGES Core MD01-2387.

\begin{tabular}{|c|c|c|c|c|c|c|c|c|c|c|c|c|}
\hline \multirow{2}{*}{$\begin{array}{l}\text { Depth } \\
\text { Interval } \\
(\mathrm{cm})\end{array}$} & \multicolumn{2}{|c|}{$161-162$} & \multicolumn{2}{|c|}{188 - 189} & \multicolumn{2}{|c|}{$350-351$} & \multicolumn{2}{|c|}{$501-502$} & \multicolumn{2}{|c|}{$667-668$} & \multicolumn{2}{|c|}{1080 - 1081} \\
\hline & $\begin{array}{l}\text { Mean } \\
(w t \%)\end{array}$ & $1 \mathrm{~s}$ & $\begin{array}{l}\text { Mean } \\
(w t \%)\end{array}$ & $1 \mathrm{~s}$ & $\begin{array}{l}\text { Mean } \\
(w t \%)\end{array}$ & $1 \mathrm{~s}$ & $\begin{array}{l}\text { Mean } \\
(w t \%)\end{array}$ & $1 \mathrm{~s}$ & $\begin{array}{l}\text { Mean } \\
(w t \%)\end{array}$ & $1 \mathrm{~s}$ & $\begin{array}{l}\text { Mean } \\
(w t \%)\end{array}$ & $1 \mathrm{~s}$ \\
\hline $\mathrm{SiO}_{2}$ & 78.73 & 0.33 & 78.79 & 0.66 & 79.53 & 0.40 & 79.73 & 0.45 & 75.27 & 0.73 & 79.31 & 0.56 \\
\hline $\mathrm{TiO}_{2}$ & 0.23 & 0.09 & 0.19 & 0.09 & 0.19 & 0.07 & 0.20 & 0.07 & 0.33 & 0.10 & 0.17 & 0.13 \\
\hline $\mathrm{Al}_{2} \mathrm{O}_{3}$ & 12.79 & 0.19 & 12.78 & 0.48 & 13.22 & 0.37 & 12.92 & 0.26 & 13.86 & 0.43 & 12.36 & 0.30 \\
\hline $\mathrm{FeO}$ & 0.88 & 0.26 & 0.43 & 0.23 & 0.82 & 0.25 & 0.98 & 0.24 & 1.50 & 0.45 & 0.57 & 0.27 \\
\hline $\mathrm{MnO}$ & 0.07 & 0.08 & 0.08 & 0.13 & 0.08 & 0.10 & 0.09 & 0.10 & 0.10 & 0.12 & 0.17 & 0.10 \\
\hline $\mathrm{MgO}$ & 0.23 & 0.04 & 0.22 & 0.05 & 0.22 & 0.03 & 0.23 & 0.04 & 0.34 & 0.06 & 0.18 & 0.02 \\
\hline $\mathrm{CaO}$ & 1.12 & 0.11 & 1.14 & 0.21 & 1.12 & 0.13 & 1.16 & 0.09 & 1.43 & 0.16 & 0.90 & 0.09 \\
\hline $\mathrm{Na}_{2} \mathrm{O}$ & 2.93 & 0.12 & 3.30 & 0.13 & 1.97 & 0.08 & 1.95 & 0.12 & 3.93 & 0.16 & 3.29 & 0.10 \\
\hline \multirow[t]{2}{*}{$\mathrm{K}_{2} \mathrm{O}$} & 3.01 & 0.13 & 3.07 & 0.18 & 2.85 & 0.23 & 2.75 & 0.14 & 3.23 & 0.12 & 3.05 & 0.10 \\
\hline & \multicolumn{2}{|l|}{$\mathrm{n}=13$} & \multicolumn{2}{|l|}{$\mathrm{n}=13$} & \multicolumn{2}{|l|}{$\mathrm{n}=10$} & \multicolumn{2}{|l|}{$\mathrm{n}=12$} & \multicolumn{2}{|l|}{$\mathrm{n}=28$} & \multicolumn{2}{|l|}{$\mathrm{n}=11$} \\
\hline $\mathrm{Na}_{2} \mathrm{O}+\mathrm{K}_{2} \mathrm{O}$ & 5.94 & 0.19 & 6.37 & 0.17 & 4.82 & 0.24 & 4.70 & 0.20 & 7.17 & 0.20 & 6.34 & 0.13 \\
\hline $\mathrm{Na}_{2} \mathrm{O} / \mathrm{K}_{2} \mathrm{O}$ & 0.98 & 0.05 & 1.08 & 0.08 & 0.70 & 0.07 & 0.71 & 0.05 & 1.22 & 0.07 & 1.08 & 0.05 \\
\hline $\begin{array}{l}\text { Isotope } \\
\text { Ratio }\end{array}$ & Mean & $2 \mathrm{~s}$ & Mean & $2 s$ & Mean & $2 \mathrm{~s}$ & Mean & $2 \mathrm{~s}$ & Mean & $2 s$ & Mean & $2 \mathrm{~s}$ \\
\hline${ }^{87} \mathrm{Sr} /{ }^{86} \mathrm{Sr}$ & 0.70388 & 0.00002 & 0.70386 & 0.00002 & n.a. & & n.a. & & 0.70420 & 0.00003 & 0.70403 & 0.00004 \\
\hline $\begin{array}{l}\text { Depth } \\
\text { Interval } \\
\text { (cm) }\end{array}$ & \multicolumn{2}{|c|}{$1120-1121$} & \multicolumn{2}{|c|}{1238 - 1239} & \multicolumn{2}{|c|}{$1444-1445$} & \multicolumn{2}{|c|}{1580 - 1581} & \multicolumn{2}{|c|}{1747 - 1748} & \multicolumn{2}{|c|}{$1822-1823$} \\
\hline $\begin{array}{l}\text { Major } \\
\text { Oxides }\end{array}$ & $\begin{array}{l}\text { Mean } \\
(w t \%)\end{array}$ & $1 \mathrm{~s}$ & $\begin{array}{l}\text { Mean } \\
(w t \%)\end{array}$ & $1 \mathrm{~s}$ & $\begin{array}{l}\text { Mean } \\
(w t \%)\end{array}$ & $1 \mathrm{~s}$ & $\begin{array}{l}\text { Mean } \\
(w t \%)\end{array}$ & $1 \mathrm{~s}$ & $\begin{array}{l}\text { Mean } \\
(w t \%)\end{array}$ & $1 \mathrm{~s}$ & $\begin{array}{l}\text { Mean } \\
(w t \%)\end{array}$ & $1 \mathrm{~s}$ \\
\hline $\mathrm{SiO}_{2}$ & 79.64 & 0.35 & 77.30 & 0.52 & 61.36 & 0.66 & 76.40 & 0.47 & 79.28 & 0.56 & 80.14 & 0.43 \\
\hline $\mathrm{TiO}_{2}$ & 0.23 & 0.09 & 0.24 & 0.09 & 0.60 & 0.16 & 0.22 & 0.12 & 0.21 & 0.10 & 0.17 & 0.10 \\
\hline $\mathrm{Al}_{2} \mathrm{O}_{3}$ & 12.97 & 0.25 & 13.07 & 0.33 & 18.15 & 0.57 & 13.73 & 0.36 & 12.12 & 0.21 & 12.85 & 0.22 \\
\hline $\mathrm{FeO}$ & 1.07 & 0.20 & 1.23 & 0.28 & 4.54 & 0.46 & 1.17 & 0.32 & 1.18 & 0.37 & 1.10 & 0.27 \\
\hline $\mathrm{MnO}$ & 0.10 & 0.12 & 0.10 & 0.12 & 0.18 & 0.12 & 0.07 & 0.08 & 0.09 & 0.11 & 0.05 & 0.09 \\
\hline $\mathrm{MgO}$ & 0.20 & 0.03 & 0.23 & 0.03 & 1.66 & 0.10 & 0.19 & 0.04 & 0.20 & 0.03 & 0.26 & 0.04 \\
\hline $\mathrm{CaO}$ & 1.04 & 0.08 & 1.15 & 0.14 & 3.91 & 0.24 & 1.04 & 0.12 & 1.23 & 0.12 & 1.40 & 0.18 \\
\hline $\mathrm{Na}_{2} \mathrm{O}$ & 1.87 & 0.31 & 3.32 & 0.32 & 4.39 & 0.15 & 3.57 & 0.13 & 3.07 & 0.29 & 1.88 & 0.16 \\
\hline \multirow[t]{2}{*}{$\mathrm{K}_{2} \mathrm{O}$} & 2.88 & 0.18 & 3.36 & 0.13 & 5.20 & 0.28 & 3.61 & 0.16 & 2.61 & 0.11 & 2.15 & 0.10 \\
\hline & \multicolumn{2}{|l|}{$\mathrm{n}=12$} & \multicolumn{2}{|l|}{$\mathrm{n}=40$} & \multicolumn{2}{|l|}{$\mathrm{n}=17$} & \multicolumn{2}{|l|}{$n=36$} & \multicolumn{2}{|l|}{$\mathrm{n}=32$} & $\mathrm{n}=11$ & \\
\hline $\mathrm{Na}_{2} \mathrm{O}+\mathrm{K}_{2} \mathrm{O}$ & 4.75 & 0.27 & 6.68 & 0.38 & 9.59 & 0.28 & 7.18 & 0.18 & 5.68 & 0.31 & 4.03 & 0.18 \\
\hline $\mathrm{Na}_{2} \mathrm{O} / \mathrm{K}_{2} \mathrm{O}$ & 0.66 & 0.12 & 0.99 & 0.10 & 0.85 & 0.06 & 0.99 & 0.06 & 1.18 & 0.12 & 0.88 & 0.09 \\
\hline $\begin{array}{l}\text { Isotope } \\
\text { Ratio }\end{array}$ & Mean. & $2 \mathrm{~s}$ & Mean & $2 \mathrm{~s}$ & Mean & $2 \mathrm{~s}$ & Mean & $2 \mathrm{~s}$ & Mean & $2 \mathrm{~s}$ & Mean & $2 \mathrm{~s}$ \\
\hline${ }^{87} \mathrm{Sr} /{ }^{86} \mathrm{Sr}$ & n.a & & 0.70405 & 0.00006 & 0.7038 & 0.00002 & 0.70401 & 0.00003 & 0.70402 & 0.00002 & 0.70390 & 0.00002 \\
\hline
\end{tabular}


Table 2. (continued).

\begin{tabular}{|c|c|c|c|c|c|c|c|c|c|c|c|c|}
\hline \multirow{2}{*}{$\begin{array}{l}\text { Depth } \\
\text { Interval } \\
(\mathbf{c m})\end{array}$} & \multicolumn{2}{|c|}{$1902-1903$} & \multicolumn{2}{|c|}{$2269-2270$} & \multicolumn{2}{|c|}{$2346-2347$} & \multicolumn{2}{|c|}{$2531-2532$} & \multicolumn{2}{|c|}{$2582-2583$} & \multicolumn{2}{|c|}{$2672-2673$} \\
\hline & $\begin{array}{l}\text { Mean } \\
(w t \%)\end{array}$ & $1 \mathrm{~s}$ & $\begin{array}{l}\text { Mean } \\
(w t \%)\end{array}$ & $1 \mathrm{~s}$ & $\begin{array}{l}\text { Mean } \\
(w t \%)\end{array}$ & $1 \mathrm{~s}$ & $\begin{array}{l}\text { Mean } \\
(w t \%)\end{array}$ & $1 \mathrm{~s}$ & $\begin{array}{l}\text { Mean } \\
(w t \%)\end{array}$ & $1 \mathrm{~s}$ & $\begin{array}{l}\text { Mean } \\
(w t \%)\end{array}$ & $1 \mathrm{~s}$ \\
\hline $\mathrm{SiO}_{2}$ & 78.96 & 0.65 & 56.74 & 1.53 & 73.58 & 0.48 & 62.19 & 1.51 & 59.27 & 1.96 & 77.99 & 0.63 \\
\hline $\mathrm{TiO}_{2}$ & 0.21 & 0.12 & 0.99 & 0.18 & 0.43 & 0.14 & 0.73 & 0.17 & 0.73 & 0.17 & 0.24 & 0.10 \\
\hline $\mathrm{Al}_{2} \mathrm{O}_{3}$ & 12.53 & 0.45 & 17.76 & 1.09 & 14.40 & 0.30 & 17.17 & 0.80 & 17.57 & 0.77 & 13.11 & 0.37 \\
\hline $\mathrm{FeO}$ & 0.65 & 0.42 & 8.35 & 0.93 & 2.44 & 0.49 & 5.53 & 0.78 & 6.90 & 0.95 & 0.70 & 0.62 \\
\hline $\mathrm{MnO}$ & 0.11 & 0.13 & 0.26 & 0.14 & 0.19 & 0.17 & 0.19 & 0.09 & 0.20 & 0.15 & 0.11 & 0.11 \\
\hline $\mathrm{MgO}$ & 0.23 & 0.04 & 2.11 & 0.73 & 0.42 & 0.06 & 1.69 & 0.17 & 2.30 & 0.50 & 0.24 & 0.04 \\
\hline $\mathrm{CaO}$ & 1.19 & 0.12 & 5.42 & 1.19 & 1.79 & 0.21 & 4.24 & 0.74 & 5.31 & 0.92 & 1.42 & 0.14 \\
\hline $\mathrm{Na}_{2} \mathrm{O}$ & 3.32 & 0.18 & 3.07 & 0.23 & 4.04 & 0.26 & 4.62 & 0.21 & 4.05 & 0.19 & 3.37 & 0.10 \\
\hline \multirow[t]{2}{*}{$\mathrm{K}_{2} \mathrm{O}$} & 2.80 & 0.19 & 5.30 & 1.02 & 2.71 & 0.08 & 3.63 & 0.37 & 3.68 & 0.51 & 2.82 & 0.11 \\
\hline & $\mathrm{n}=9$ & & $\mathrm{n}=10$ & & $\mathrm{n}=7$ & & $\mathrm{n}=10$ & & $\mathrm{n}=13$ & & $\mathrm{n}=7$ & \\
\hline $\mathrm{Na}_{2} \mathrm{O}+\mathrm{K}_{2} \mathrm{O}$ & 6.12 & 0.18 & 8.37 & 1.14 & 6.75 & 0.30 & 8.26 & 0.50 & 7.73 & 0.57 & 6.19 & 0.20 \\
\hline $\mathrm{Na}_{2} \mathrm{O} / \mathrm{K}_{2} \mathrm{O}$ & 1.19 & 0.12 & 0.60 & 0.11 & 1.49 & 0.09 & 1.28 & 0.14 & 1.12 & 0.15 & 1.19 & 0.03 \\
\hline $\begin{array}{l}\text { Isotope } \\
\text { Ratio }\end{array}$ & Mean & $2 \mathrm{~s}$ & Mean & $2 \mathrm{~s}$ & Mean & $2 \mathrm{~s}$ & Mean & $2 \mathrm{~s}$ & Mean & $2 \mathrm{~s}$ & Mean & $2 \mathrm{~s}$ \\
\hline${ }^{87} \mathrm{Sr} /{ }^{86} \mathrm{Sr}$ & 0.70389 & 0.00003 & 0.70376 & 0.00002 & 0.70414 & 0.00002 & 0.70387 & 0.00002 & 0.70383 & 0.00002 & 0.70393 & 0.00006 \\
\hline
\end{tabular}

\begin{tabular}{|c|c|c|c|c|c|c|c|c|}
\hline \multirow{2}{*}{$\begin{array}{l}\text { Depth } \\
\text { Interval } \\
(\mathrm{cm})\end{array}$} & \multicolumn{2}{|c|}{$2839-2840$} & \multicolumn{2}{|c|}{$2913-2914$} & \multicolumn{2}{|c|}{$2933-2934$} & \multicolumn{2}{|c|}{$3019-3020$} \\
\hline & $\begin{array}{l}\text { Mean } \\
(w t \%)\end{array}$ & $1 \mathrm{~s}$ & $\begin{array}{l}\text { Mean } \\
(w t \%)\end{array}$ & $1 \mathrm{~s}$ & $\begin{array}{l}\text { Mean } \\
(w t \%)\end{array}$ & $1 \mathrm{~s}$ & $\begin{array}{l}\text { Mean } \\
(w t \%)\end{array}$ & $1 \mathrm{~s}$ \\
\hline $\mathrm{SiO}_{2}$ & 58.98 & 0.79 & 76.05 & 1.02 & 78.34 & 0.93 & 53.37 & 1.84 \\
\hline $\mathrm{TiO}_{2}$ & 0.83 & 0.18 & 0.36 & 0.10 & 0.18 & 0.12 & 1.86 & 0.46 \\
\hline $\mathrm{Al}_{2} \mathrm{O}_{3}$ & 17.03 & 0.94 & 13.85 & 0.43 & 12.96 & 0.50 & 15.18 & 0.50 \\
\hline $\mathrm{FeO}$ & 6.49 & 0.55 & 1.86 & 0.51 & 0.92 & 0.19 & 9.71 & 0.80 \\
\hline $\mathrm{MnO}$ & 0.24 & 0.15 & 0.13 & 0.12 & 0.07 & 0.07 & 0.15 & 0.10 \\
\hline $\mathrm{MgO}$ & 2.42 & 0.42 & 0.27 & 0.03 & 0.26 & 0.10 & 5.67 & 0.47 \\
\hline $\mathrm{CaO}$ & 5.72 & 0.47 & 1.31 & 0.11 & 1.36 & 0.25 & 10.01 & 0.81 \\
\hline $\mathrm{Na}_{2} \mathrm{O}$ & 3.94 & 0.18 & 2.93 & 0.97 & 3.16 & 0.27 & 3.37 & 0.34 \\
\hline \multirow[t]{2}{*}{$\mathrm{K}_{2} \mathrm{O}$} & 4.34 & 0.17 & 3.24 & 0.25 & 2.74 & 0.22 & 0.69 & 0.41 \\
\hline & \multicolumn{2}{|l|}{$\mathrm{n}=7$} & \multicolumn{2}{|l|}{$\mathrm{n}=23$} & \multicolumn{2}{|l|}{$\mathrm{n}=7$} & \multicolumn{2}{|l|}{$\mathrm{n}=29$} \\
\hline $\mathrm{Na}_{2} \mathrm{O}+\mathrm{K}_{2} \mathrm{O}$ & 8.28 & 0.34 & 6.17 & 1.19 & 5.91 & 0.31 & 4.06 & 0.74 \\
\hline $\mathrm{Na}_{2} \mathrm{O} / \mathrm{K}_{2} \mathrm{O}$ & 0.91 & 0.02 & 0.89 & 0.27 & 1.16 & 0.14 & 6.50 & 2.89 \\
\hline $\begin{array}{l}\text { Isotope } \\
\text { Ratio }\end{array}$ & Mean & $2 \mathrm{~s}$ & Mean & $2 \mathrm{~s}$ & Mean & $2 \mathrm{~s}$ & Mean & $2 \mathrm{~s}$ \\
\hline${ }^{87} \mathrm{Sr} /{ }^{86} \mathrm{Sr}$ & 0.70384 & 0.00002 & 0.70418 & 0.00002 & 0.70399 & 0.00002 & 0.70383 & 0.00002 \\
\hline
\end{tabular}

Note: $s=$ standard deviation; $n$. $a .=$ not analyzed. 


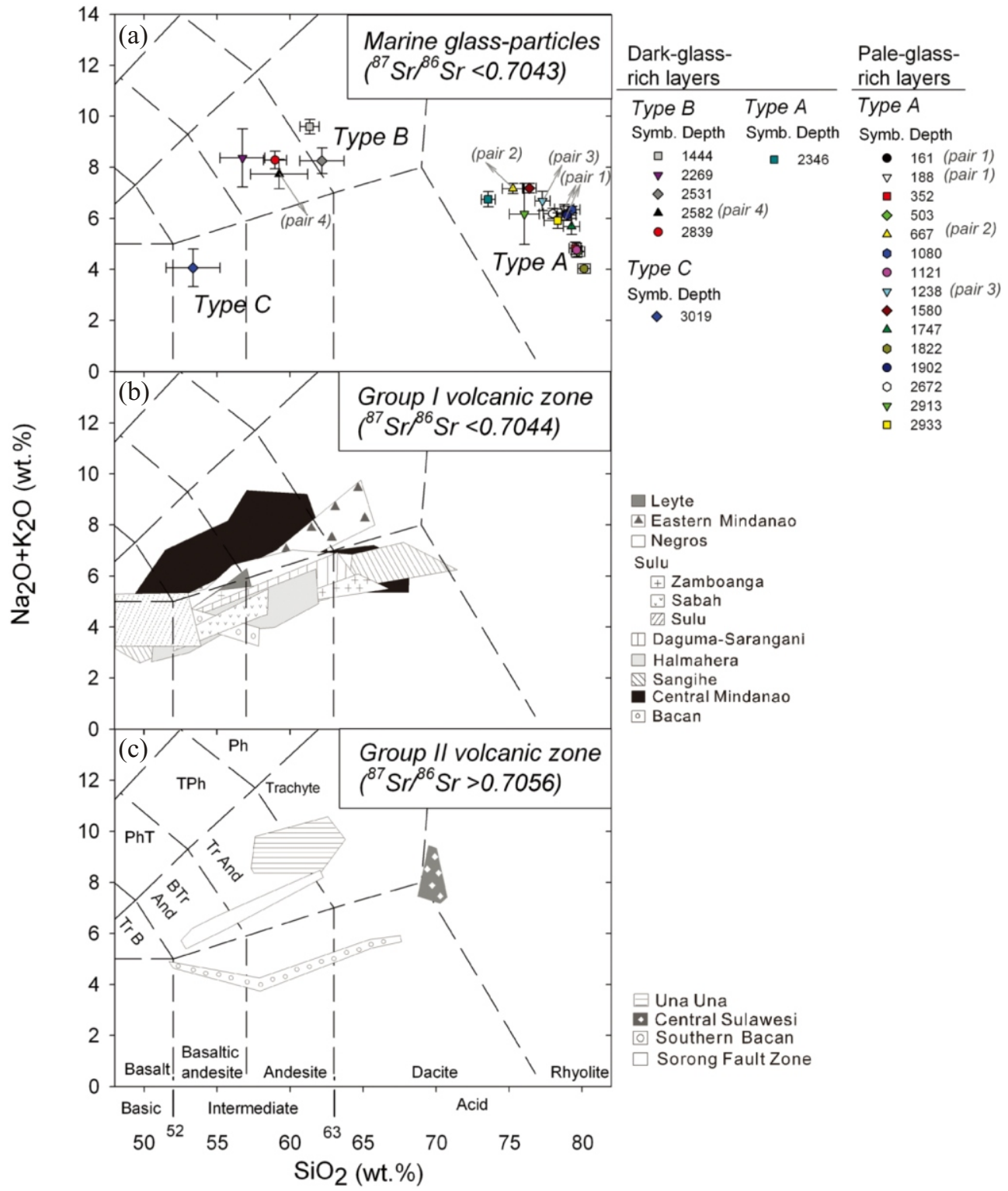

Fig. 3. Total alkalis content vs. $\mathrm{SiO}_{2}$ plot demonstrating: (a) three compositional types of the 22 analyzed glass-rich layers (Types A, B, and C), and the compositional fields for (b) Group I volcanic zone and (c) Group II volcanic zone. Glass data were obtained in this study; data on the rock types of each volcanic province in the two groups were collected from literature (e.g., Elburg and Foden 1999; Sajona et al. 2000a, b; Polvé et al. 2001; Castillo et al. 2002; Solidum et al. 2003). Nomenclature fields follow Le Maitre et al. (1989), with abbreviations: PhT, phonotephrite; TPh, tephri-phonolite; $\mathrm{Ph}$, phonolite; TrB, trachybasalt; BTrAnd, basaltic trachyandesite; TrAnd, trachyandesite. For the description of the four pairs in (a), please refer to the text.

currents. Pubellier et al. (1991) discussed the relationships of diverse tephra sources and prevailing winds for the CSB. The present wind pattern in the troposphere (Tanaka et al. 2004) indicates that, during winter, the southern Philippine arcs and to a minor extent the arcs of northeastern Indonesia (Group I volcanic zone, Fig. 1) could act as sources for the tephra layers of CSB. South of the Equator, north-directed winds could transport tephra from the northeastern Indonesia (Group II volcanic zone, Fig. 1) across the Equatorial zone up to the CSB. During July, most of the air masses move northward and would disperse ash from the Group I volcanic zone (Fig. 1). Assuming that the paleo-wind patterns around the CSB are similar to the present ones, then, all the Quaternary volcanic provinces surrounding the CSB can be considered to be potential sources of the marine tephra layers found in Core MD01-2387.

The geochemical characteristics of ash particles may enable the marine tephra sequence at Site MD01-2387 to be correlated with that of other deep-sea coring sites. These characteristics may also help in associating the individual tephra layer with specific volcanic source area. The geo- 


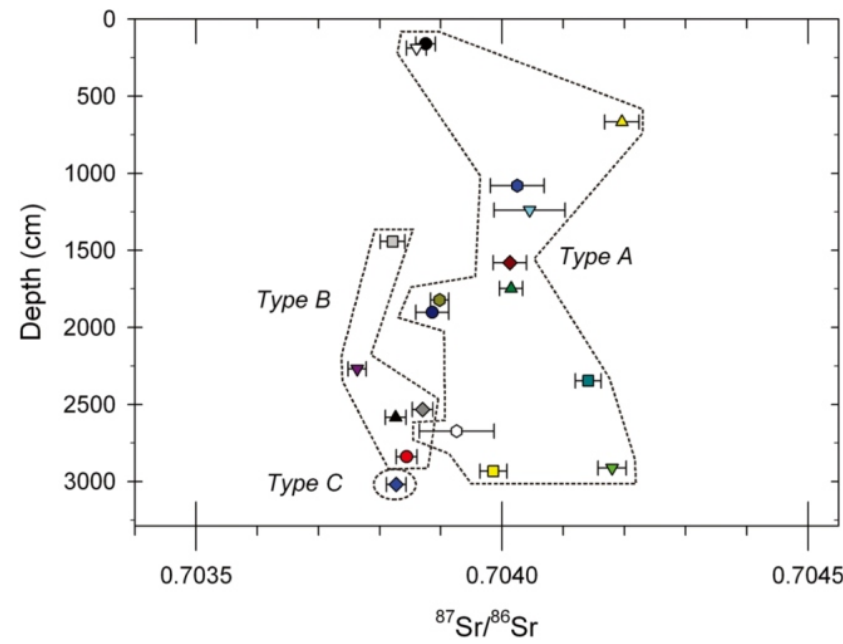

Fig. 4. The Sr isotopic ratios vs. stratigraphic depths for the 19 analyzed glass-rich layers. The legends used here are identical to that in Fig. 3a.

chemistry of particles from the tephra layers at Site MD012387 are discussed below in terms of the chronology of sediment accumulation (tephrochronology), and from where the tephra particles were injected (tephra source).

\subsection{Tephrochronology}

The depth at Site MD01-2387 is very close to the carbonate compensation depth (CCD), thus calcareous nannofossils in sediments were poorly preserved (Bassinot and Baltzer 2002) due to serve dissolution of carbonate. This inherited poor carbonate preservation incurs a difficult situation for establishing a chronological scheme using calcareous plankton biostratigraphy and marine oxygen isotope stratigraphy. Fortunately, at the nearby Site ODP 767, sediments in the upper $50 \mathrm{~m}$ are composed of hemipelagic claystone to silt with interbedded tephra layers and rare calcareous turbidite layers (Silver and Rangin 1990; Betzler et al. 1991). Although nannofossils are not abundant, probably because of dissolution, the calcareous nannofossils biostratigraphy of Hole $767 \mathrm{~B}$ along with the magnetostratigraphy was reported by Shyu and Muller (1991) and Shyu et al. (1991) (Fig. 5a). Some tephra layers at Hole ODP 767 were dated (Desprairies et al. 1991) by using biostratigraphic markers. Geochemical data of glass particles in eight tephra layers of Hole ODP 767 B were reported (Desprairies et al. 1991; Pouclet et al. 1991) (Fig. 5a). Correlation of the geochemical characteristics of glass particles from Hole ODP 767 with those from Core MD01-2387 enables several tephra layers to be key markers in estimating the stratigraphic age of IMAGES Site MD01-2387.

A comparison of the major element contents of glass particles at both sites enables identification of four correlated pairs of tephra layers (Fig. 5b). Samples at depths of (1) 161 and $188 \mathrm{~cm}$; (2) $667 \mathrm{~cm}$; (3) $1238 \mathrm{~cm}$; and (4) $2582 \mathrm{~cm}$ at Site MD01-2387 can be correlated to that at (1) $74 \mathrm{~cm}$; (2) 368 and $377 \mathrm{~cm}$; (3) $874 \mathrm{~cm}$; and (4) $1836 \mathrm{~cm}$ in ODP Site 767 , respectively (Table 3, Fig. 6). The depths of the four pairs of tephra layers in the two cores show a good linear relationship $\left(\mathrm{R}^{2}=0.99\right)$, indicating a constant ratio between the sedimentation rates at two sites. Extrapolating the linearity between the four tephra layer pairs (Fig. 7), the oldest occurrence of Emiliania huxleyi, $275 \mathrm{ka}$, is expected to be at $2668 \mathrm{~cm}$ in Core MD01-2387. Based upon this depth and the age, subtracting ash layer thickness (Table 1) and assuming a constant sedimentation rate for the remaining sediments throughout the core, the sedimentation rate at Site MD01-2387 is estimated to be around $9.3 \mathrm{~cm} \mathrm{kyr}^{-1}$, while the age for the core bottom is estimated to be ca. $338 \mathrm{ka}$.

Estimated ages for the depths of 1975, 1800, and $1444 \mathrm{~cm}$ in Core MD01-2387 were ca. 204, 187, and $151 \mathrm{ka}$, respectively (Fig. 7). The age at 204 ka represents the beginning of the decreasing proportion of crystal-rich layers (Table 1, Figs. 2b, 8b). The age at 151 ka represents the last occurrence of the Type B dark-glass-rich layers (Table 1, Figs. 2b, 8b). At the depths of $<1800 \mathrm{~cm}(<187 \mathrm{ka})$, the ash layers decreased up-sequence (Figs. 2b, 8a).

\subsection{Tephra Sources}

\subsubsection{Crystal-Rich Layers (27 layers)}

The distance between Site MD01-2387 and source volcanoes for the crystal-rich layers (up to 27 layers) can be approximated using fallout observations. Given the discrepant densities of the mineral crystals and the vesicular glass particles, their dispersion behavior and transport distance in air have been modeled using ash dispersion and sedimentation (e.g., Bursik et al. 1992; Bonadonna and Phillips 2003). In principle, lighter particles should have lower terminal velocity and be dispersed over a longer distance than heavier particles, given similar particle sizes. The relative abundance of heavier particles in ash-fallout deposit should thus decrease with increased transport distance (Fierstein and Nathenson 1992).

Two well-documented examples of these processes were the 1980 eruption of Mt. St. Helen (VEI = 4) (Carey and Sigurdsson 1982) and the 1991 eruption of Mt. Pinatubo $(\mathrm{VEI}=5)$ (Wiesner et al. 2004). Observations of particle transport from these two plinian eruptions (Fig. 9) show two distinctive features: first, glass particles tend to become the dominant constituent (> 60 wt.\%) as downwind distance exceeds ca. $300 \mathrm{~km}$ from the source; and second, the relative crystal abundance is lower than ca. $40 \mathrm{wt} . \%$ when the downwind distance exceeds ca. $300 \mathrm{~km}$. Thus, as shown in Fig. 9, assuming that the eruption intensity and magnitude were smaller than or equal to VEI of 5, the source volcanoes of the crystal-rich layers should lie within roughly $250 \mathrm{~km}$ of Site MD01-2387 (the circle enclosing the marked area in Fig. 1). Furthermore, considering the wind direction during 
(a) Hole ODP 767B

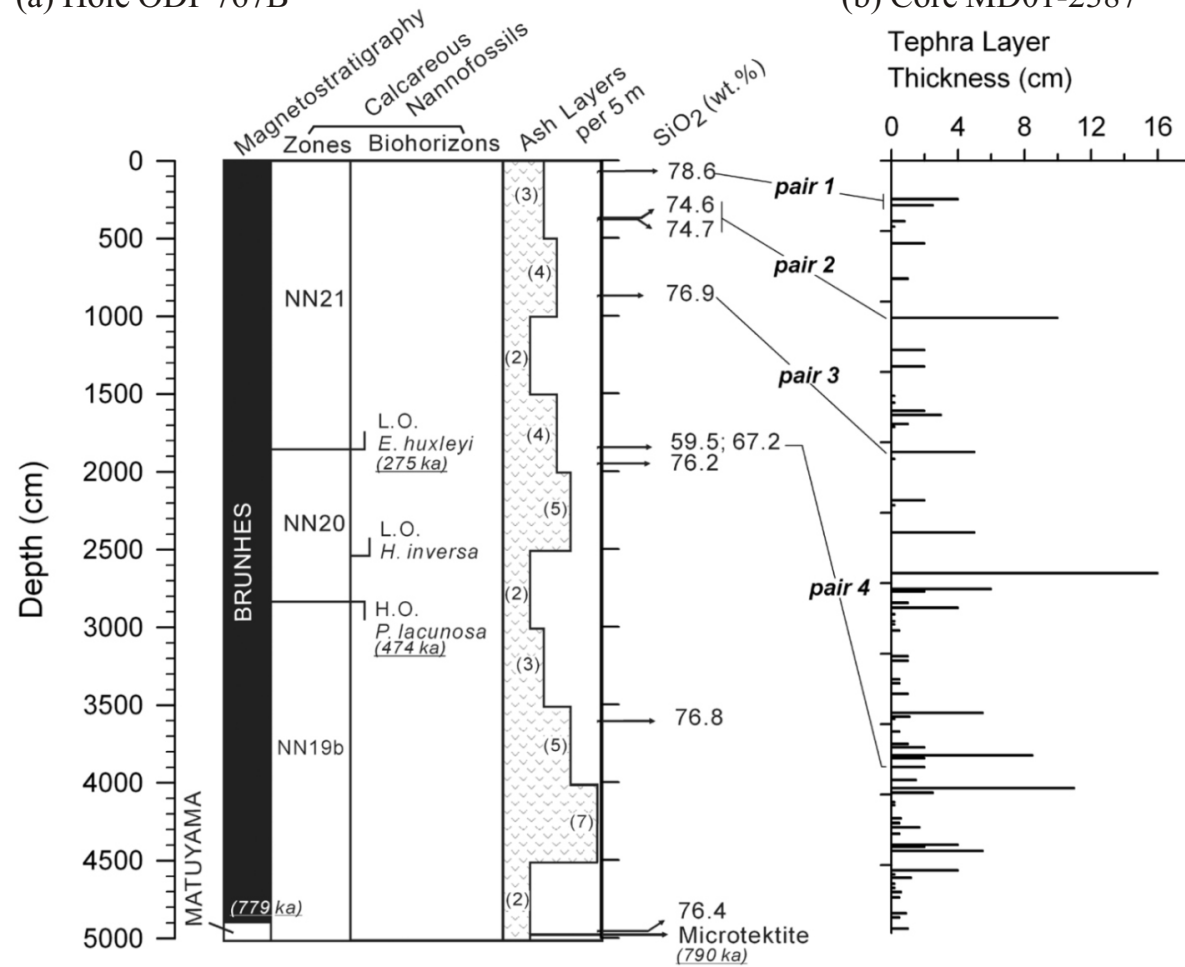

(790 ka) (b) Core MD01-2387

Ash Layers per $3 \mathrm{~m}$

Fig. 5. (a) Chronologic framework for the upper portion sediments in Hole ODP $767 \mathrm{~B}$, as well as the ash layers histogram and the eight ash layers with published chemical data $\left(\mathrm{SiO}_{2}\right.$ wt.\%) of glass. (b) Tephra layer thickness and number plotted against depth in Core MD01-2387. Data sources for Hole ODP 767 B: Shipboard Scientific Party 1990; Desprairies et al. 1991; Pouclet et al. 1991; Shyu and Muller 1991; Shyu et al. 1991; Schneider et al. 1992; Oda et al. 2000. L.O. = lowest occurrence; H.O. = highest occurrence. For definitions of the layers, marked pairs 1 - 4, please refer to the text.

Table 3. Chemical composition of 10 remarked glass particles in the IMAGES and ODP cores displayed by four pairs of correlated ash layers.

\begin{tabular}{|c|c|c|c|c|c|c|c|c|c|c|}
\hline \multirow{3}{*}{$\begin{array}{l}\text { Pair } \\
\text { Core } \\
\text { Site }\end{array}$} & \multicolumn{3}{|c|}{1} & \multicolumn{3}{|c|}{2} & \multicolumn{2}{|c|}{3} & \multicolumn{2}{|l|}{4} \\
\hline & \multirow{2}{*}{\multicolumn{2}{|c|}{$\begin{array}{c}\text { IMAGES } \\
\text { MD01-2387 }\end{array}$}} & \multirow{2}{*}{$\begin{array}{l}\text { ODP } \\
767 A\end{array}$} & \multirow{2}{*}{$\begin{array}{c}\text { IMAGES } \\
\text { MD01-2387 }\end{array}$} & \multicolumn{2}{|c|}{ ODP } & \multirow{2}{*}{$\begin{array}{c}\text { IMAGES } \\
\text { MD01-2387 }\end{array}$} & \multirow{2}{*}{$\begin{array}{l}\text { ODP } \\
767 B\end{array}$} & \multirow{2}{*}{$\begin{array}{c}\text { IMAGES } \\
\text { MD01-2387 }\end{array}$} & \multirow{2}{*}{$\begin{array}{l}\text { ODP } \\
767 B\end{array}$} \\
\hline & & & & & $767 \mathrm{~A}$ & 767B & & & & \\
\hline $\begin{array}{l}\text { Depth } \\
\text { Interval } \\
(\mathrm{cm})\end{array}$ & $161-162$ & $188-189$ & $74-76$ & $667-668$ & $\begin{array}{c}368- \\
370\end{array}$ & $\begin{array}{c}377- \\
379\end{array}$ & $1238-1239$ & $874-876$ & $2582-2583$ & $\begin{array}{c}1836 \\
1838\end{array}$ \\
\hline $\mathrm{SiO}_{2}$ & $78.73 \pm 0.33$ & $78.79 \pm 0.66$ & 78.62 & $75.27 \pm 0.73$ & 74.64 & 74.70 & $77.30 \pm 0.52$ & 76.90 & $59.27 \pm 1.96$ & 59.52 \\
\hline $\mathrm{TiO}_{2}$ & $0.23 \pm 0.09$ & $0.19 \pm 0.09$ & 0.09 & $0.33 \pm 0.10$ & 0.36 & 0.32 & $0.24 \pm 0.09$ & 0.19 & $0.73 \pm 0.17$ & 0.87 \\
\hline $\mathrm{Al}_{2} \mathrm{O}_{3}$ & $12.79 \pm 0.19$ & $12.78 \pm 0.48$ & 12.56 & $13.86 \pm 0.43$ & 14.06 & 13.85 & $13.07 \pm 0.33$ & 13.26 & $17.57 \pm 0.77$ & 17.73 \\
\hline $\mathrm{FeO}_{\mathrm{T}}$ & $0.88 \pm 0.26$ & $0.43 \pm 0.23$ & 0.85 & $1.50 \pm 0.45$ & 1.58 & 1.55 & $1.23 \pm 0.28$ & 1.07 & $6.90 \pm 0.95$ & 6.02 \\
\hline $\mathrm{MnO}$ & $0.07 \pm 0.08$ & $0.08 \pm 0.13$ & 0.13 & $0.10 \pm 0.12$ & 0.05 & 0.15 & $0.10 \pm 0.12$ & 0.04 & $0.20 \pm 0.15$ & 0.18 \\
\hline $\mathrm{MgO}$ & $0.23 \pm 0.04$ & $0.22 \pm 0.05$ & 0.22 & $0.34 \pm 0.06$ & 0.39 & 0.39 & $0.23 \pm 0.03$ & 0.22 & $2.30 \pm 0.50$ & 2.04 \\
\hline $\mathrm{CaO}$ & $1.12 \pm 0.11$ & $1.14 \pm 0.21$ & 0.98 & $1.43 \pm 0.16$ & 1.36 & 1.31 & $1.15 \pm 0.14$ & 1.28 & $5.31 \pm 0.92$ & 4.84 \\
\hline $\mathrm{Na}_{2} \mathrm{O}$ & $2.93 \pm 0.12$ & $3.30 \pm 0.13$ & 3.24 & $3.93 \pm 0.16$ & 4.13 & 4.27 & $3.32 \pm 0.32$ & 3.93 & $4.05 \pm 0.19$ & 4.90 \\
\hline $\mathrm{K}_{2} \mathrm{O}$ & $3.01 \pm 0.13$ & $3.07 \pm 0.18$ & 3.30 & $3.23 \pm 0.12$ & 3.44 & 3.47 & $3.36 \pm 0.13$ & 3.11 & $3.68 \pm 0.51$ & 3.90 \\
\hline $\mathrm{Na}_{2} \mathrm{O}+\mathrm{K}_{2} \mathrm{O}$ & $5.94 \pm 0.19$ & $6.37 \pm 0.17$ & 6.55 & $7.17 \pm 0.20$ & 7.56 & 7.73 & $6.68 \pm 0.38$ & 7.05 & $7.73 \pm 0.57$ & 8.80 \\
\hline $\mathrm{Na}_{2} \mathrm{O} / \mathrm{K}_{2} \mathrm{O}$ & $0.98 \pm 0.05$ & $1.08 \pm 0.08$ & 0.98 & $1.22 \pm 0.07$ & 1.20 & 1.23 & $0.99 \pm 0.10$ & 1.26 & $1.12 \pm 0.15$ & 1.26 \\
\hline Data Source & this $\mathrm{s}$ & tudy & (1) & this study & & & this study & (2) & this study & (1) \\
\hline
\end{tabular}

Note: Major elements are normalized to 100 wt. \% and all iron are converted to $\mathrm{FeO}_{T}: \mathrm{FeO}_{T}=0.899 \times \mathrm{Fe}_{2} \mathrm{O}_{3}+\mathrm{FeO}$.

Data source: (1) Pouclet et al. (1991); (2) Desprairies et al. (1991). 
the eruption, prospective source volcanoes for the crystalrich layers should be located within the volcanic provinces in the northern (Sulu Arc), through the northeastern (Daguma-Sarangani Arc), and the eastern (northern Sangihe Arc) sides of Site MD01-2387 (Fig. 1). All prospective source volcanic centers for the crystal-rich layers appear to be restricted to the northwestern part of the Group I volcanic zone (Fig. 1). Another notable phenomenon is that the number of crystal-rich layers decreases significantly after $240 \mathrm{ka}$ (Fig. 8b). This indicates that explosive activities in the arcs of northwestern Mindanao-Molucca Sea collision zone have declined during the past 240 kyrs.

\subsubsection{Glass-Rich Layer (22 Layers)}

The tephra source can be traced by correlating the chemical and isotopic characteristics of the glass particles with those of the late Quaternary volcanic rocks surrounding the CSB. The Sr isotopic ratios of glass particles, ranging from 0.70376 to 0.70420 (Figs. 3a, 4), suggest that the glass particles were not eruptive products of the volcanic provinces in the Group II volcanic zone (>0.7056) (Fig. 3c) in the southern Celebes Sea and Molucca Sea (Fig. 1). Moreover, the isotopic ratios of the glass particles were comparable with those of the volcanic provinces from the Group I volcanic

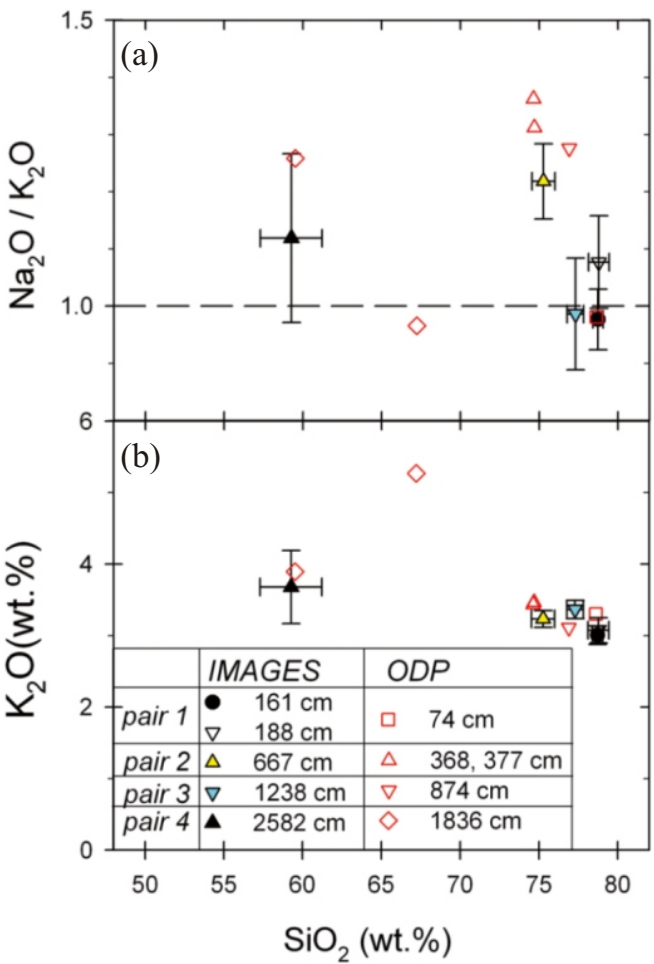

Fig. 6. Four correlative ash layer pairs of IMAGES Site MD01-2387 and ODP Site 767 in the (a) $\mathrm{Na}_{2} \mathrm{O} / \mathrm{K}_{2} \mathrm{O}$ vs. $\mathrm{SiO}_{2}$ and (b) $\mathrm{K}_{2} \mathrm{O}$ vs. $\mathrm{SiO}_{2}$ diagrams. The bar length or box side represents one standard deviation.

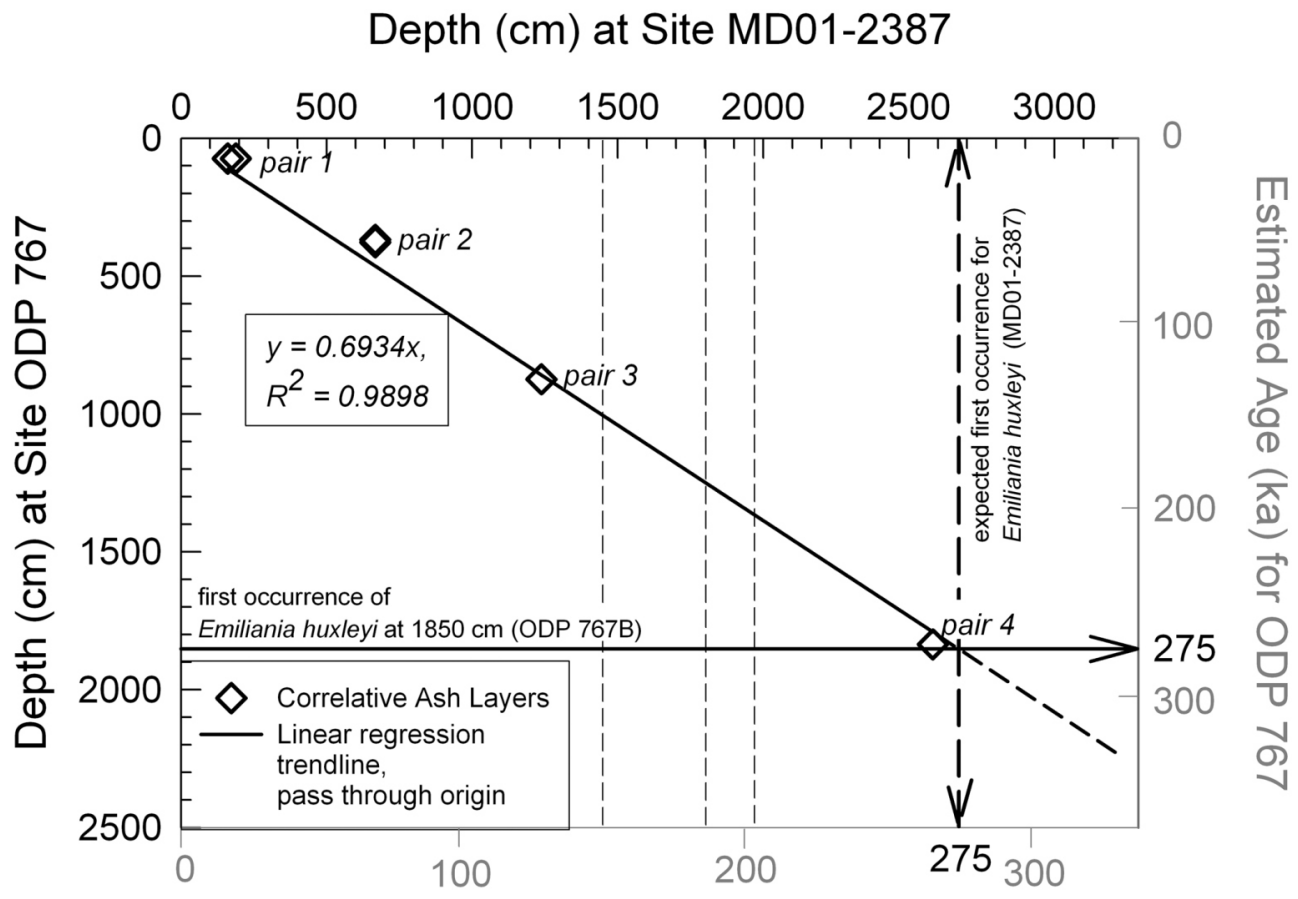

Estimated Age (ka) for MD01-2387

Fig. 7. Stratigraphic depth correlation for four pairs of correlative ash layers in IMAGES Site MD01-2387 and ODP Site 767. Based upon the depth of the first occurrence of Emiliania huxleyi at Site ODP 767 (Shyu et al. 1991), its corresponding depth in Site MD01-2387 can be estimated by the regression trend line $\left(\mathrm{R}^{2}=0.99\right)$. The age of four depths $(3288,1975,1800$, and $1444 \mathrm{~cm})$ at Site MD01-2387 can be estimated $(338,204,187$, and $151 \mathrm{ka}$ ) by subtracting the thickness of ash layers in this core and assuming a constant relationship between the sedimentation rates of the two sites. 
(a)

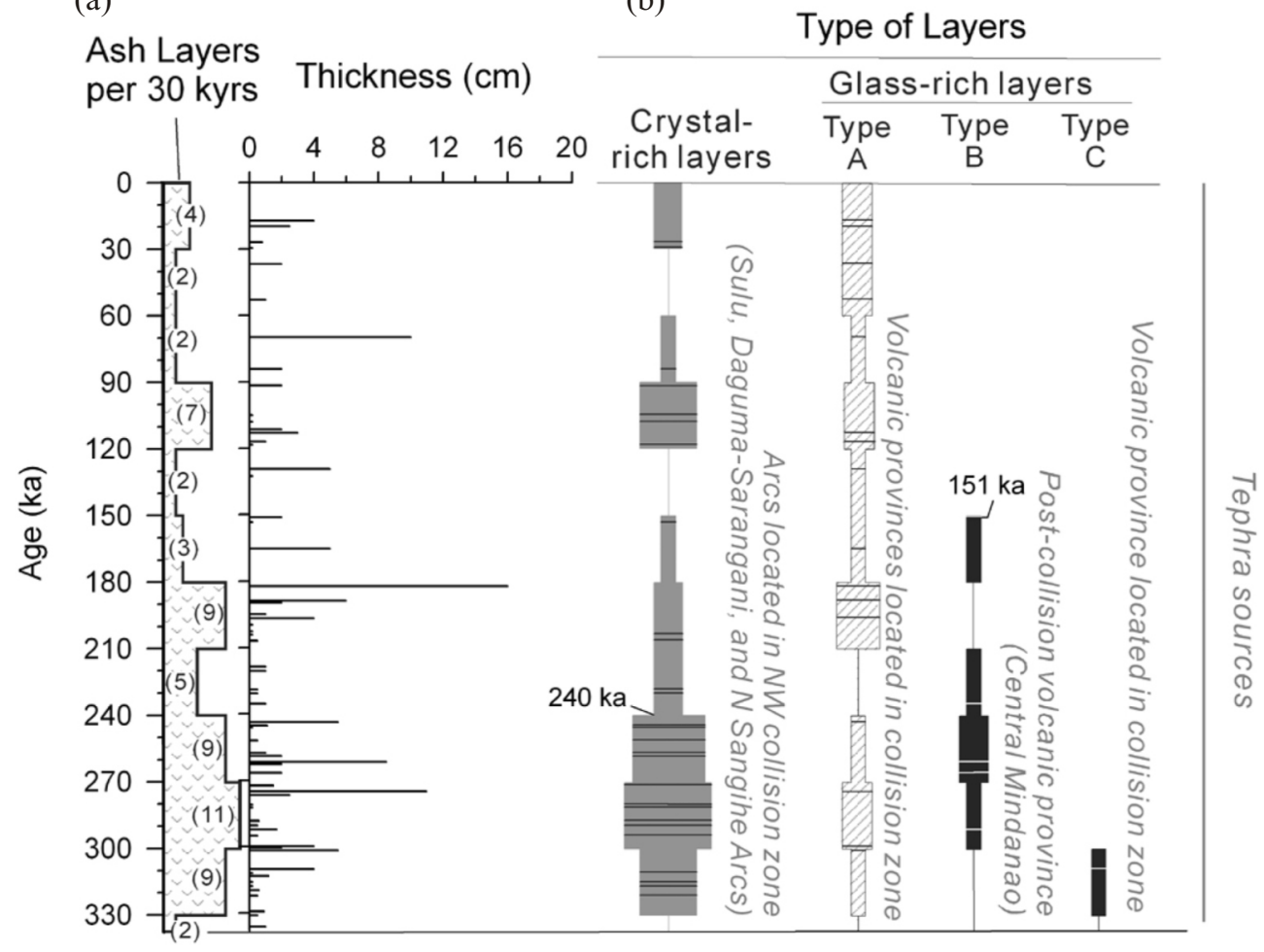

Fig. 8. (a) Histograms depict number of ash layers and layer thickness as well as (b) bar charts representing the relative portion of various types of tephra layers plotted against estimated age. Tephra sources for each type of layers are marked to the right side. The estimated age is based on the plot of Fig. 7. Data of ash layers are based on Table 1. The bar length represents the number of ash layers per 30 kyrs. Solid lines in black or white color within the boxes mark the position of ash layers.

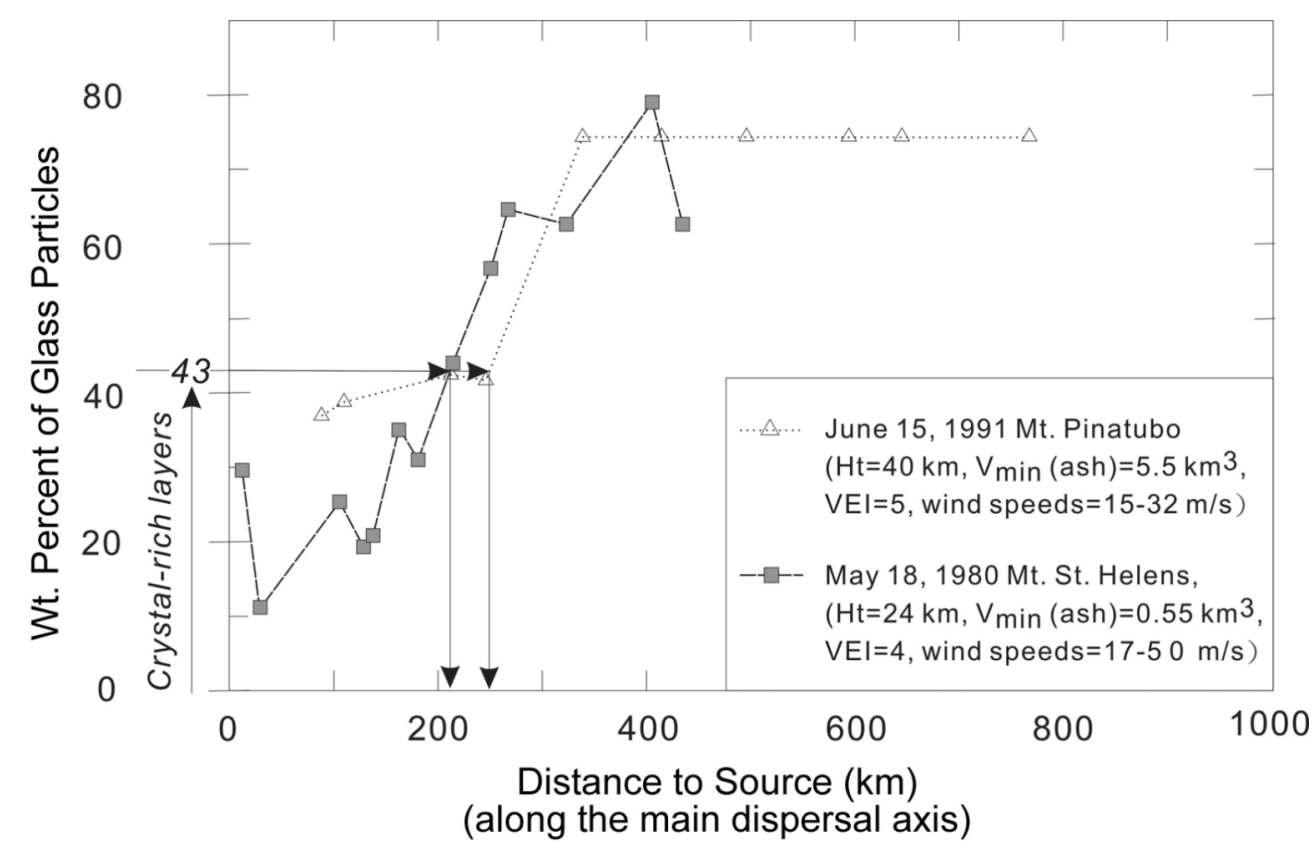

Fig. 9. Constitutions of two well-investigated ash deposits varying with transport distances. Maximum transported distances for the crystal-rich layers of Core MD01-2387 can be delineated by the estimated fraction of glass particles less than $43 \mathrm{wt} . \%$, based on the nature of their constitution (mineral crystals: $>50$ vol. $\%$, density of plagioclase and pyroxene: $2.62-3.96 \mathrm{~g} \mathrm{~cm}^{-3}$, density of glass pumice $(63-500 \mu \mathrm{m}): 2.0-1.0 \mathrm{~g} \mathrm{~cm}{ }^{-3}$ by Bonadonna and Phillips 2003). Data sources for the two ash-fallout deposits are from Carey and Sigurdsson (1982) and Wiesner et al. (2004), respectively. 
zone $(<0.7044)$ (Fig. 3b). Thus, the volcanic provinces in the Group I volcanic zone, located in the MindanaoMolucca Sea collision zone, were the most likely tephra source areas for these glass-rich layers (Fig. 8b).

\section{Type A Glass-Rich Layers}

Type A glass particles, with 3.4 to 7.6 wt. $\% \mathrm{Na}_{2} \mathrm{O}+\mathrm{K}_{2} \mathrm{O}$ at $>73.0$ wt. $\% \mathrm{SiO}_{2}$, are rhyolite (Fig. 3a). The total alkalis content $\left(\mathrm{Na}_{2} \mathrm{O}+\mathrm{K}_{2} \mathrm{O}\right)$ vs. $\mathrm{SiO}_{2}$ plot of these glass particles (Fig. 3a), with its relatively higher $\mathrm{SiO}_{2}$, fall in the extrapolation area of the composition fields of lavas and pyroclastic flows of the Group I volcanic zone (Fig. 3b). The lavas and pyroclastic flows, from the Group I volcanic zone, are basalt to andesite or dacite. Nevertheless, it should be noted that lava/ash with higher $\mathrm{SiO}_{2}$ content could have been produced from the eruption center, and therefore yielding, by fractionation crystalization, glasses with chemical properties similar to those of the Type A glass particles.

\section{Type B Glass-Rich Layers}

The glass particles of Type B glass-rich layers, with a composition $>6.1$ wt. $\% \mathrm{Na}_{2} \mathrm{O}+\mathrm{K}_{2} \mathrm{O}$ at $54.7-64.7$ wt. $\%$ $\mathrm{SiO}_{2}$, range in composition from trachyandesite to trachyte (Fig. 3a). The compositions of Type B glass particles (Fig. 3a) overlap directly one of the composition fields of volcanic rocks in the Central Mindanao Area (Fig. 3b). Furthermore, volcanic rocks in the Central Mindanao Area (Fig. 3b), possessing intermediate $\mathrm{SiO}_{2}$ and higher total alkalis content, can be easily discerned from other volcanic provinces of Group I. Thus, particles of the Type B glass-rich layers are recognized as products of the Central Mindanao Area. The tephrostratigraphy shows that the Type B glass-rich layers are not found in the intervals younger than ca. $151 \mathrm{ka}$ (Fig. 8b), suggesting that the explosive volcanism with shoshonitic composition from the Central Mindanao Area ceased ca. $151 \mathrm{ka}$.

\section{Type C Glass-Rich Layer}

The Type $\mathrm{C}$ glass particles, with compositions of 3.1 5.2 wt. $\% \mathrm{Na}_{2} \mathrm{O}+\mathrm{K}_{2} \mathrm{O}$ and 50.6 - 55.8 wt. $\% \mathrm{SiO}_{2}$, are basalt or basaltic andesite (Fig. 3a). The Type $\mathrm{C}$ glass particles fall in the compositional fields of volcanic rocks of the Group I volcanic zone (Fig. 3b) and can not be linked to a specific volcanic province by their major element characteristic alone.

\subsection{Volcanic Activity}

The marine tephrostratigraphy of Core MD01-2387 records the episodicity of large explosive volcanism of the Mindanao-Molucca Sea collision zone (Group I volcanic zone, Fig. 1) over the past 338 kyrs (Fig. 8) and shows that after ca. $180 \mathrm{ka}$, explosive volcanism from specific volcanic areas of the collision zone (the northwestern collision area and the post-collision volcanic area) appears to have undergone a decline. This is inferred from source tracking the two types of ash layers, i.e., the crystal-rich layers and the darkglass-rich layers of Type B composition (Fig. 8b). The former significantly attenuates in the shallower sequence of the core ( $<1975 \mathrm{~cm}$ in depth) while the latter disappears entirely in the upper sequence of the core $(<1444 \mathrm{~cm}$ in depth) (Table 1 and Fig. 2b). The decline or absence of these ash layers indicates two important traits of the explosive eruption history of the region: fewer large explosive eruptions since ca. $240 \mathrm{ka}$, and the termination of large explosive eruptions with shoshonitic composition after ca. $151 \mathrm{ka}$ (Fig. 8b). The former occurred in arcs of the northwestern collision area including the Sulu, Daguma-Sarangani, and northern Sangihe Arcs, while the latter is found in the post-collision volcanic area (the Central Mindanao Area). Thus, the marine tephrostratigraphy of Core MD01-2387 documents better the explosive volcanic history for the youngest part (late Middle Pleistocene) of the last major volcanically active period (since ca. $2.5 \mathrm{Ma}$ ) of the volcanic provinces in the Mindanao-Molucca Sea collision zone (Group I volcanic zone).

At issue is whether the volcanic activity inferred from the marine tephrostratigraphy of MD01-2387 being consistent with previous onland reports. Two onland volcanic provinces of this collision zone (Group I volcanic zone) are related to the tephra records with the upward attenuation in the core. They are: (1) the Central Mindanao Area and (2) the northwestern Mindanao-Molucca Sea collision area. The first province is considered to be the source area for the Type B dark-glass-rich layers. The second province is suggested to be the source for the crystal-rich layers. A detailed comparison between previous onland studies of the two provinces and the current tephrostratigraphic record is given in the following. In addition, the previous onland report for discussing the volcanic activity of the southern Molucca Sea and Celebes Sea (Group II volcanic zone) is also mentioned.

\subsubsection{Central Mindanao Area}

The onland investigation of the Central Mindanao Area by Sajona et al. (1994) shows that potassic and shoshonitic magmatisms occurred frequently between $0.8-0.36 \mathrm{Ma}$. No evidence of potassic and shoshonitic magmatisms in the Central Mindanao Area during the past $0.36 \mathrm{~m}$.y. was found. The feature of extinction of shoshonitic magmatism is observed in many post-collision regions such as western Anatolia in Turkey and the Roman volcanic province in Italy (Sajona et al. 1994, 1997). Core MD01-2387 shows five layers of glass particles composed of shoshonite and high-K se- 
ries (Type B layers) (Fig. 3a) correlatable to the volcanic province of the Central Mindanao Area (Fig. 3b). Based upon the fact that these Type B layers were deposited prior to ca. $151 \mathrm{ka}$ (Table 1, Fig. 8b), we suggested that the cessation of the shoshonitic magmatism occurred much younger than what suggested by previous onland studies which reported an extinction age of $0.36 \pm 0.12 \mathrm{Ma}$ using K-Ar dating (Sajona et al. 1994, 1997, 2000b).

\subsubsection{Northwestern Mindanao-Molucca Sea Collision Area}

The northwestern Mindanao-Molucca Sea collision area includes three volcanic provinces: (1) the Sulu Arc, (2) the Daguma-Sarangani Arc in the complete-collision zone, and (3) the northern Sangihe Arc in the transition-collision zone (Fig. 1). Based on the dating results of the volcanic rocks in the transition-collision zone from the southern end of Daguma-Sarangani Arc down to Sangihe Island (Fig. 1), Morrice et al. (1983) found that the termination of the Quaternary volcanism in the northern Sangihe Arc was a result of the continuous welding of the two arcs in the MindanaoMolucca Sea area. This termination front has migrated from the southern end of Mindanao Island to Sangihe Island (Morrice et al. 1983). The southward migration of the termination of volcanism in the transition-collision zone may explain the attenuation of the crystal-rich layers in the deep sea basin since ca. 240 ka (Fig. 8b).

\subsubsection{The Southern Molucca Sea and Celebes Sea}

Given the present wind pattern (Tanaka et al. 2004), the winter wind would disperse the eruptive products of the volcanic provinces in the southern-southeastern side of the CSB (Group II volcanic zone) to the studied site. Based upon the constituent of 27 crystal-rich layers and the geochemical characteristics of 22 glass-rich layers of Core MD01-2387, however, the volcanic provinces in Group II volcanic zone are not a main source for the tephra layers of the core.

The MD01-2387 deep-sea record agrees with the report of onland investigation in Group II volcanic zone. Onland investigation offered features of explosive eruptions in three provinces (Volcano Una Una, Central Sulawesi Area, and Bacan Island). First, for the area of the Gorontalo Gulf, the volcanic activity was virtually extinct during the Quaternary (Katili et al. 1963), with the exception of Volcano Una Una, which commenced eruption in historical time (Katili et al. 1963; Katili and Sudradjat 1984). However, the strongest explosive event in recorded history occurred from April to October 1898 (Katili et al. 1963; Katili and Sudradjat 1984). The ashes released from this event were blown to the west, falling on Borneo (Fig. 1), and did not reach Site MD01-2387.
Secondly, for the Central Sulawesi Area, the youngest volcanic rock, Barupu Tuff, is reported to be of an age between 0.61 - 0.55 Ma by K-Ar dating (Priadi et al. 1994), that is older than the estimated age for the core bottom of MD01-2387. Finally, for the Bacan Island, the volcanism record of onland investigations was reported with long-term extinction of volcanism after ca. $2 \mathrm{Ma}$ (Baker and Malaihollo 1996). Therefore, the onland reports for the feature of explosive eruptions of these three provinces support the explanation of why the tephra particles of 22 layers in Core MD01-2387 (Fig. 3a) do not match geochemically with the volcanics in Group II volcanic zone (Fig. 3c).

\subsubsection{Spatial and Temporal Distribution}

The marine tephrostratigraphy of Core MD01-2387 documents a detailed explosive volcanic history for the youngest part $(<338 \mathrm{ka})$ of the last major volcanically active pe$\operatorname{riod}(<2.5 \mathrm{Ma})$ in the Mindanao-Molucca Sea collision zone (Group I volcanic zone) and shows that the explosive volcanic activity in the Mindanao-Molucca Sea collision zone has been declining since ca. $180 \mathrm{ka}$. Compiling the results of Core MD01-2387 and previous onland reports and other deep-sea tephra records, spatial and temporal distribution of the active volcanic area surrounding the Celebes Sea can be illustrated (Fig. 10). Following the closure of the Molucca Sea, after $2.5 \mathrm{Ma}$ (Fig. 10a), volcanism was active in many volcanic provinces in Mindanao-Molucca Sea collision zone, either related to the subduction systems or associated with the post-collision magmatism (Pubellier et al. 1991; Sajona et al. 1997). After $240 \mathrm{ka}$ (Fig. 10b), the explosive volcanism in northwestern Mindanao-Molucca Sea collision zone tended to be less frequent than what occurred during 240 $338 \mathrm{ka}$. In the post-collision volcanic province (i.e., Central Mindanao), volcanism with potassic-shoshonitic composition was active starting around 0.8 Ma (Sajona et al. 1994), but became extinct approximately ca. $151 \mathrm{ka}$.

\section{CONCLUSIONS}

Tephrostratigraphy of piston Core MD01-2387 shows that ash layers accumulated densely in the lower part of the sequence (ca. 180 - $338 \mathrm{ka}$ ). The up-sequence decline in the proportion of ash layers implies that the explosive volcanic activity in the Mindanao-Molucca Sea collision zone has been declining since ca. $180 \mathrm{ka}$. The record provides new evidence in suggesting that the shoshonitic magmatism in Central Mindanao became extinct approximately ca. $151 \mathrm{ka}$, much younger than what reported by previous onland studies.

Acknowledgements The authors are indebted to Prof. M. T. Chen of the National Taiwan Ocean University and the scientific party as well as crew members of the Marion 

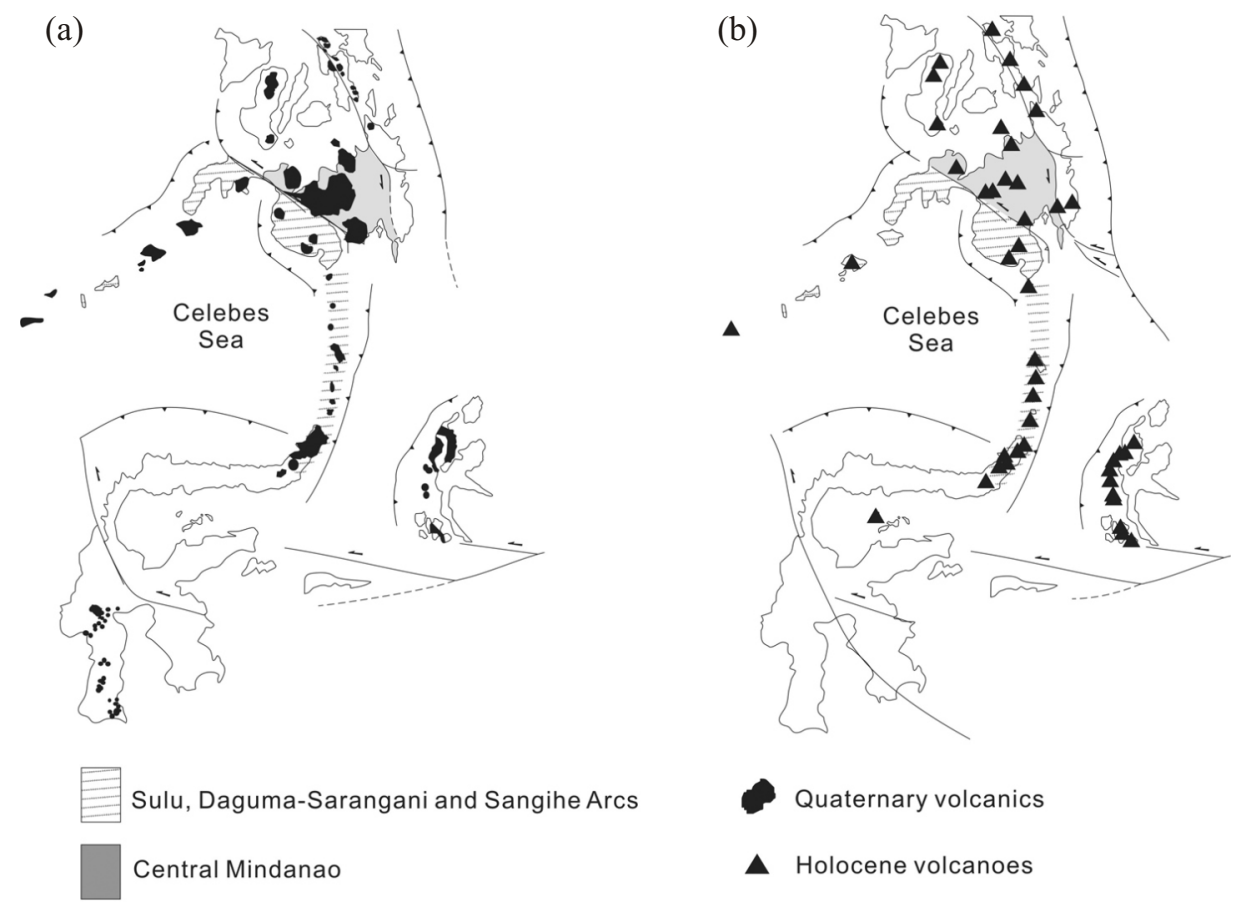

Fig. 10. Spatial and temporal distribution of the active volcanic areas surrounding the Celebes Sea: (a) Quaternary volcanics erupted before ca. 240 ka and (b) volcanoes erupting after ca. $240 \mathrm{ka}$. Data sources: geographic outline - Quebral et al. (1996), Hall (1997), and Hinschberger et al. (2005); volcanic ages - Sajona et al. (1994), Baker and Malaihollo (1996), Polvé et al. (1997), Sajona et al. (1997), and Elburg and Foden (1999); and Holocene active volcanoes - Simkin and Siebert (1994).

Dufresne for coring in the Celebes Sea on the IMAGESVII cruise. We wish to thank Dr. C. Laj for kindly providing the samples of Core MD01-2387 and Dr. T. Q. Lee and Dr. W. L. Wang for transporting the samples to Taiwan. We gratefully acknowledge helpful discussions with Prof. K. Y. Wei and Prof. T. F. Yang in National Taiwan University at several stages in the study progress. This research was supported by the National Science Council of the Republic of China to C. H. Chen (NSC 94-2116-M-001-009 and NSC 95-2116-001-004). The detailed and constructive reviews of two anonymous reviewers as well as suggestions by editor Dr. Kuo-Yen Wei improved the paper and are gratefully acknowledged.

\section{REFERENCES}

Baker, S. and J. Malaihollo, 1996: Dating of Neogene igneous rocks in the Halmahera region: Arc initiation and development. In: Hall, R. and D. J. Blundell (Eds.), Tectonic Evolution of Southeast Asia, Geological Social, London, Special Publications, 106, 499-509.

Bassinot, F. and A. Baltzer, 2002: WEPAMA Cruise MD 122/ IMAGES VII, Leg 1, Leg 2. Institut Polaire Français, Les rapports des campagnes à la mer, $453 \mathrm{pp}$.

Betzler, C., A. J. Nederbragt, and G. J. Nichols, 1991: Significance of turbidites at site 767 (Celebes sea) and site 768 (Sulu sea). In: Silver, E. A., C. Rangin, M. T. von
Breymann, and Leg 124 Scientific Party (Eds.), Proceedings of the Ocean Drilling Program, Scientific Results, 124, 431-446.

Bonadonna, C. and J. C. Phillips, 2003: Sedimentation from strong volcanic plumes. J. Geophys. Res., 108, 2340-2368, doi: 10.1029/2002JB002034. [Link]

Bursik, M. I., R. S. J. Sparks, J. S. Gilbert, and S. N. Carey, 1992: Sedimentation of tephra by volcanic plumes: I. Theory and comparison with fallout from the Fogo A plinian deposit, Sao Miguel (Azores). Bull. Volcanol., 54, 329344, doi: 10.1007/BF00301486. [Link]

Carey, S. N. and H. Sigurdsson, 1982: Influence of particle aggregation on deposition of distal tephra from the May 18, 1980, eruption of Mount St. Helens volcano. J. Geophys. Res., 87, 7061-7072, doi: 10.1029/JB087iB08p07061. [Link]

Castillo, P. R., R. U. Solidum, and R. S. Punongbayan, 2002: Origin of high field strength element enrichment in the Sulu Arc, southern Philippines, revisited. Geology, 30, 707-710, doi: 10.1130/0091-7613(2002)030<0707:OOHFSE $>$ 2.0.CO;2. [Link]

Delfin, F. G. Jr., C. G. Newhall, M. L. Martinez, N. D. Salonga, F. E. B. Bayon, D. Trimble, and R. Solidum, 1997: Geological, ${ }^{14} \mathrm{C}$, and historical evidence for a $17^{\text {th }}$ century eruption of Parker Volcano, Mindanao, Philippines. $J$. Geol. Soc. Phil., 52, 25-42.

Desprairies, A., M. Rivière, and M. Pubellier, 1991: Diagenetic 
evolution of Neogene volcanic ashes (Celebes and Sulu seas). In: Silver, E. A., C. Rangin, M. T. von Breymann, and Leg 124 Scientific Party (Eds.), Proceedings of the Ocean Drilling Program, Scientific Results, 124, 489-506.

Elburg, M. and J. Foden, 1998: Temporal changes in arc magma geochemistry, Northern Sulawesi, Indonesia. Earth Planet. Sci. Lett., 163, 381-398, doi: 10.1016/S0012-821X(98) 00143-5. [Link]

Elburg, M. A. and J. Foden, 1999: Sources for magmatism in Central Sulawesi: Geochemical and $\mathrm{Sr}-\mathrm{Nd}-\mathrm{Pb}$ isotopic constraints. Chem. Geol., 156, 67-93, doi: 10.1016/S00092541(98)00175-2. [Link]

Fierstein, J. and M. Nathenson, 1992: Another look at the calculation of fallout tephra volumes. Bull. Volcanol., 54, 156-167, doi: 10.1007/BF00278005. [Link]

Hall, R., 1997: Cenozoic plate tectonic reconstructions of SE Asia. In: Fraser, A. J., S. J. Matthews, and R. W. Murphy (Eds.), Petroleum Geology of Southeast Asia, Geological Society of London, Special Publication, 126, 11-23.

Hinschberger, F., J. A. Malod, J. P. Réhault, M. Villeneuve, J. Y. Royer, and S. Burhanuddin, 2005: Late Cenozoic geodynamic evolution of eastern Indonesia. Tectonophysics, 404, 91-118, doi: 10.1016/j.tecto.2005.05.005. [Link]

Katili, J. A. and A. Sudradjat, 1984: The devastating 1983 eruption of Colo volcano, Una-Una Island, central Sulawesi, Indonesia. Geol. Jahrb., 75, 27-47.

Katili, J. A., L. Kartaadiputra, and Surio, 1963: Magma type and tectonic position of the Una-Una Island, Indonesia. Bull. Volcanol., 26, 431-454, doi: 10.1007/BF02597303. [Link]

Kopp, C., E. R. Flueh, and S. Neben, 1999: Rupture and accretion of the Celebes Sea crust related to the North-Sulawesi subduction: Combined interpretation of reflection and refraction seismic measurements. J. Geodyn., 27, 309-325, doi: 10.1016/S0264-3707(98)00004-0. [Link]

Le Maitre, R. W., P. Bateman, A. Dudek, J. Keller, J. Lameyre, M. J. Le Bas, P. A. Sabine, R. Schmid, H. Sorensen, A. Streckeisen, A. R. Woolley, and B. Zanettin, 1989: A Classification of Igneous Rocks and Glossary of Terms, Blackwell, Oxford, 1-193.

Macpherson, C. G., E. J. Forde, R. Hall, and M. F. Thirlwall, 2003: Geochemical evolution of magmatism in an arc-arc collision: The Halmahera and Sangihe arcs, eastern Indonesia. In: Larter, R. D. and P. T. Leat (Eds.), Intra-oceanic Subduction Systems: Tectonic and Magmatic Processes, Geological Society of London, Special Publication, 219, 207-220.

Moore, G. F. and E. A. Silver, 1983: Collision processes in the Northern Molucca Sea. In: Hayes, D. E. (Ed.), The Tectonic and Geologic Evolution of Southeast Asian Seas and Islands: Part 2, AGU Geophys. Monogr. Ser., 27, 360-372.

Morrice, M. G., P. A. Jezek, J. B. Gill, D. J. Whitford, and M. Monoarfa, 1983: An introduction to the Sangihe arc: Volcanism accompanying arc-arc collision in the Molucca Sea, Indonesia. J. Volcanol. Geotherm. Res., 19, 135-165, doi: 10.1016/0377-0273(83)90129-4. [Link]

Morris, J. D., P. A. Jezek, S. R. Hart, and J. B. Gill, 1983: The Halmahera arc, Molucca sea collision zone, Indonesia: A geochemical survey. In: Hayes, D. E. (Ed.), The Tectonic and Geologic Evolution of Southeast Asian Seas and Islands: Part 2, AGU Geophys. Monogr. Ser., 27, 373-387.

Moss, S. J., A. Carter, S. Baker, and A. J. Hurford, 1998: A Late Oligocene tectono-volcanic event in East Kalimantan and the implications for tectonics and sedimentation in Borneo. J. Geol. Soc., 155, 177-192, doi: 10.1144/gsjgs.155. 1.0177. [Link]

Oda, H., H. Shibuya, and V. Hsu, 2000: Palaeomagnetic records of Brunhes/Matuyama polarity transition from ODP Leg 124 (Celebes and Sulu Seas). Geophys. J. Int., 142, 319338, doi: 10.1046/j.1365-246x.2000.00130.x. [Link]

Polvé, M., R. C. Maury, H. Bellon, C. Rangin, B. Priadi, S. Yuwono, J. L. Joron, and R. S. Atmadja, 1997: Magmatic evolution of Sulawesi (Indonesia): Constraints on the Cenozoic geodynamic history of the Sundaland active margin. Tectonophysics, 272, 69-92, doi: 10.1016/S00401951(96)00276-4. [Link]

Polvé, M., R. C. Maury, P. Vidal, B. Priadi, H. Bellon, R. Soeria-Atmadja, J.-L. Joron, and J. Cotten, 2001: Melting of lower continental crust in a young post-collision setting: A geochemical study of Plio-Quaternary acidic magmatism from central Sulawesi (Indonesia). Bull. Soc. Géol. Fr., 172, 333-342, doi: 10.2113/172.3.333. [Link]

Pouclet, A., M. Pubellier, and P. Spadea, 1991: Volcanic ash from Celebes and Sulu Sea basins off the Philippines (Leg 124): Petrography and geochemistry. In: Silver, E. A., C. Rangin, M. T. von Breymann, and Leg 124 Scientific Party (Eds.), Proceedings of the Ocean Drilling Program, Scientific Results, 124, 467-487.

Priadi, B., M. Polve, R. C. Maury, H. Bellon, R. SoeriaAtmadja, J. L. Joron, and J. Cotton, 1994: Tertiary and Quaternary magmatism in Central Sulawesi: Chronological and petrological constraints. J. Southeast Asian Earth Sci., 9, 81-93, doi: 10.1016/0743-9547(94)90067-1. [Link]

Pubellier, M., P. Spadea, A. Pouclet, R. Solidum, A. Desprairies, and H. Cambray, 1991: Correlations of tephras in Celebes and Sulu Sea basins: Constraints on geodynamics. In: Silver, E. A., C. Rangin, M. T. von Breymann, and Leg 124 Scientific Party (Eds.), Proceedings of the Ocean Drilling Program, Scientific Results, 124, 459-465.

Pubellier, M., A. G. Bader, C. Rangin, B. Deffontaines, and R. Quebral, 1999: Upper plate deformation induced by subduction of a volcanic arc: The Snellius plateau (Molluca sea, and Indonesia and Mindanao, Philippines). Tectonophysics, 304, 345-368, doi: 10.1016/S0040-1951(98) 00300-X. [Link]

Rangin, C., 1991: The Philippine mobile belt: A complex plate boundary. J. Southeast Asian Earth Sci., 6, 209-220, doi: 10.1016/0743-9547(91)90068-9. [Link]

Sajona, F. G. and R. C. Maury, 1998: Association of adakites with gold and copper mineralization in the Philippines. $C$. 
R. Acad. Sci. Ser. IIA Earth Planet. Sci., 326, 27-34, doi: 10.1016/ S1251-8050(97)83200-4. [Link]

Sajona, F. G., H. Bellon, R. C. Maury, M. Pubellier, J. Cotten, and C. Rangin, 1994: Magmatic response to abrupt changes in geodynamic settings: Pliocene-Quaternary calc-alkaline lavas and $\mathrm{Nb}$ enriched basalts of Leyte and Mindanao (Philippines). Tectonophysics, 237, 47-72, doi: 10.1016/ 0040-1951(94)90158-9. [Link]

Sajona, F. G., H. Bellon, R. C. Maury, M. Pubellier, R. D. Quebral, J. Cotten, F. E. Bayon, E. Pagado, and P. Pamatian, 1997: Tertiary and Quaternary magmatism in Mindanao and Leyte (Philippines): Geochronology, geochemistry and tectonic setting. J. Asian Earth Sci., 15, 121153, doi: 10.1016/S0743-9547(97)00002-0. [Link]

Sajona, F. G., R. C. Maury, G. Prouteau, J. Cotten, P. Schiano, H. Bellon, and L. Fontaine, 2000a: Slab melt as metasomatic agent in island arc magma mantle sources, Negros and Batan (Philippines). Isl. Arc, 9, 472-486, doi: 10.1046/ j.1440-1738.2000.00295.x. [Link]

Sajona, F. G., R. C. Maury, M. Pubellier, J. Leterrier, H. Bellon, and J. Cotten, 2000b: Magmatic source enrichment by slab-derived melts in a young post-collision setting, central Mindanao (Philippines). Lithos, 54, 173-206, doi: 10.1016/S0024-4937(00)00019-0. [Link]

Schneider, D., D. Kent, and G. Mello, 1992: A detailed chronology of the Australasian impact event, the BrunhesMatuyama geomagnetic polarity reversal, and global climatic change. Earth Planet. Sci. Lett., 111, 395-405, doi: 10.1016/0012-821X(92)90192-X. [Link]

Shipboard Scientific Party, 1990: Site 767. In: Rangin, C., E. Silver, M. T. von Breymann, and Leg 124 Scientific Party (Eds.), Proceedings of the Ocean Drilling Program, Scientific Results., 124, 121-193.

Shyu, J. P. and C. Muller, 1991: Calcareous nannofossil biostratigraphy of the Celebes and Sulu Seas. In: Silver, E. A., C. Rangin, M. T. von Breymann, and Leg 124 Scientific Party (Eds.), Proceedings of the Ocean Drilling Program, Scientific Results., 124, 133-157.

Shyu, J. P., D. Merrill, V. Hsu, M. A. Kaminski, C. M. Müller, A. J. Nederbragt, R. P. Scherer, and H. Shibuya, 1991: Biostratigraphic and magnetostratigraphic synthesis of the Celebes and Sulu Seas. In: Silver, E. A., C. Rangin, M. T. von Breymann, and Leg 124 Scientific Party (Eds.), Proceedings of the Ocean Drilling Program, Scientific Results., 124, 11-35.

Silver, E. A. and C. Rangin, 1990: Leg 124 of the ocean drilling program. Geophys. Res. Lett., 17, 2059-2060, doi: 10.1029/ GL017i011p02059. [Link]

Simkin, T. and L. Siebert, 1994: Volcanoes of the World, $2^{\text {nd }}$ edition: Geoscience Press in association with the Smithsonian Institution Global Volcanism Program, Tucson AZ, 368 pp.

Solidum, R. U., P. R. Castillo, and J. W. Hawkins, 2003: Geochemistry of lavas from Negros Arc, west central Philippines: Insights into the contribution from the subducting slab. Geochem. Geophys. Geosyst., 4, 9008, doi: 10.1029/ 2003GC000513. [Link]

Tanaka, H. L., N. Ishizaki, and A. Kitoh, 2004: Trend and interannual variability of Walker, monsoon and Hadley circulations defined by velocity potential in the upper troposphere. Tellus, Series A, 56, 250-269, doi: 10.1111/ j.1600-0870.2004.00049.x. [Link]

Taylor, S. R., R. Arculus, M. R. Perfit, and R. Johnson, 1981: Island arc basalts. In: Basaltic Volcanism in the Terrestrial Planets (Basaltic Volcanism Study Project), Pergamon Press, New York, 193-213.

Tongkul, F., 1991: Tectonic evolution of Sabah, Malaysia. J. Southeast Asian Earth Sci., 6, 395-405, doi: 10.1016/ 0743-9547(91)90084-B. [Link]

Wichmann, A., 1902: Der Vulkan der Insel Una-Una (Nanguna) in Busen von Tomini, Celebes. J. Ger. Geol. Soc., 54, 144-158.

Wiesner, M. G., Y. Wang, and L. Zheng, 1995: Fallout of volcanic ash to the deep South China Sea induced by the 1991 eruption of Mount Pinatubo (Philippines). Geology, 23, 885-888, doi: 10.1130/0091-7613(1995)023<0885: FOVATT $>2.3 . \mathrm{CO} ; 2$. [Link]

Wiesner, M. G., A. Wetzel, S. G. Catane, E. L. Listanco, and H. T. Mirabueno, 2004: Grain size, areal thickness distribution and controls on sedimentation of the 1991 Mount Pinatubo tephra layer in the South China Sea. Bull. Volcanol., 66, 226-242, doi: 10.1007/s00445-003-0306-x. [Link] 